Supporting Information

\title{
Palladium-Catalyzed Alkylarylation of Acrylamides with Unactivated Alkyl Halides
}

Hua Wang, Li-Na Guo, and Xin-Hua Duan*

Department of Chemistry, School of Science and MOE Key Laboratory for

Nonequilibrium Synthesis and Modulation of Condensed Matter, Xi'an Jiaotong

\author{
University, Xi'an 710049, China \\ duanxh@mail.xjtu.edu.cn
}

\section{Table of Contents}

Investigation of the Reaction Mechanism $\quad$ S2

Isotope Labeling Experiment $\quad$ S5

$\begin{array}{ll}\text { Reference } & \text { S8 }\end{array}$

${ }^{1} \mathrm{H}$ NMR and ${ }^{13} \mathrm{C}$ NMR Spectra of Products $3 \quad$ S9

${ }^{1} \mathrm{H}$ NMR and ${ }^{13} \mathrm{C}$ NMR Spectra of Products $4 \quad$ S28

${ }^{1} \mathrm{H}$ NMR and ${ }^{13} \mathrm{C}$ NMR Spectra of Products 6a $\quad$ S46

${ }^{1} \mathrm{H}$ NMR and ${ }^{13} \mathrm{C}$ NMR Spectra of Products 8a and 8b $\quad$ S47 


\section{Investigation of the Reaction Mechanism}

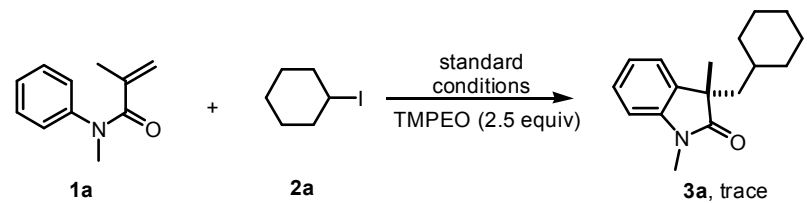

When 2.5 equiv of TEMPO was added to the reaction of 1a with $\mathbf{2 a}$ under the standard conditions, only trace amount of desired product 3a was detected, along with $83 \%$ of 1 a recovered. The result indicates that a radical intermediate is probably involved in the catalytic cycle of the reaction.

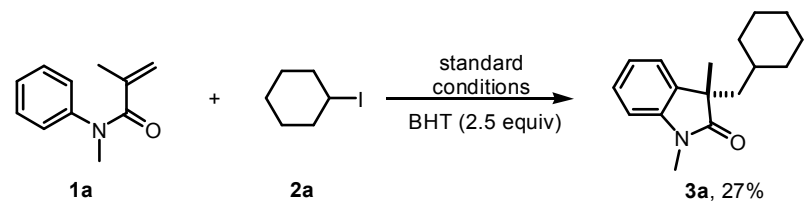

When 2.5 equiv of BHT was added to the reaction of $\mathbf{1 a}$ with $\mathbf{2 a}$ under the standard conditions, only $27 \%$ yield of desired product $3 \mathbf{a}$ was isolated. The result indicates that a radical intermediate is probably involved in the catalytic cycle of the reaction. 


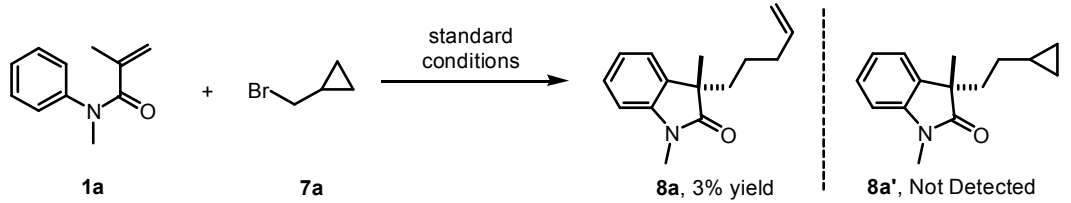

When cyclopropylmethyl bromide 7a was treated with 1a under the standard conditions, only 3\% yield of the ring-opening product $8 \mathbf{a}$ was obtained due to the low conversion of 1a. In this case, 1a was recovered in $91 \%$. No cyclopropylethyl substituted product $\mathbf{8} \mathbf{a}^{\prime}$ was detected in this reaction.

1,3-dimethyl-3-pent-4-enyl-1,3-dihydro-indol-2-one (8a): ${ }^{1} \mathrm{H}$ NMR $(400 \mathrm{MHz}$, $\left.\mathrm{CDCl}_{3}\right): \delta=7.29-7.25(\mathrm{~m}, 1 \mathrm{H}), 7.17(\mathrm{~d}, J=7.2 \mathrm{~Hz}, 1 \mathrm{H}), 7.12-7.05(\mathrm{~m}, 1 \mathrm{H}), 6.84(\mathrm{~d}, J$ $=7.6 \mathrm{~Hz}, 1 \mathrm{H}), 5.70-5.60(\mathrm{~m}, 1 \mathrm{H}), 5.23-5.22(\mathrm{~m}, 1 \mathrm{H}), 4.93-4.87(\mathrm{~m}, 1 \mathrm{H}), 3.22(\mathrm{~s}, 3 \mathrm{H})$, 1.95-1.70 (m, 2H), 1.66-1.53 (m, 2H), 1.35 (s, 3H), 0.94-0.83 (m, 2H); ${ }^{13} \mathrm{C}$ NMR (100 $\left.\mathrm{MHz}, \mathrm{CDCl}_{3}\right): \delta=180.7,143.3,138.2,134.1,127.6,122.4,114.7,107.9,48.3,38.0$, 33.7, 26.1, $23.7 \mathrm{ppm}$; The NMR spectrum of 8a displayed relatively impurities in the aliphatic and aromatic region due to other inseparable byproducts. HRMS (ESI) calcd for $\mathrm{C}_{15} \mathrm{H}_{19} \mathrm{NNaO}[\mathrm{M}+\mathrm{Na}]^{+} 252.1359$, found 252.1362 .

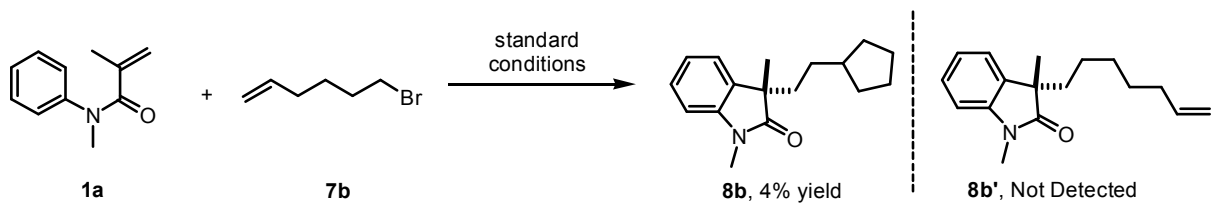

When 6-bromo-1-hexene $\mathbf{7 b}$ was treated with 1a under the standard conditions, only $4 \%$ yield of the cyclopentylethyl substituted oxindole $\mathbf{8 b}$ was obtained due to the low conversion of 1a. In this case, 1a was recovered in $87 \%$. No heptenyl substituted product $\mathbf{8} \mathbf{b}^{\prime}$ was detected in this reaction.

3-(2-Cyclopentyl-ethyl)-1,3-dimethyl-1,3-dihydro-indol-2-one (8b): ${ }^{1} \mathrm{H}$ NMR (400 $\left.\mathrm{MHz}, \mathrm{CDCl}_{3}\right): \delta=7.29-7.25(\mathrm{~m}, 1 \mathrm{H}), 7.17(\mathrm{~d}, J=7.2 \mathrm{~Hz}, 1 \mathrm{H}), 7.07(\mathrm{t}, J=7.2 \mathrm{~Hz}$, $1 \mathrm{H}), 6.84(\mathrm{~d}, J=7.6 \mathrm{~Hz}, 1 \mathrm{H}), 3.22(\mathrm{~s}, 3 \mathrm{H}), 1.91-1.86(\mathrm{~m}, 2 \mathrm{H}), 1.78-1.57(\mathrm{~m}, 3 \mathrm{H})$, 1.53-1.41 (m, 3H), $1.35(\mathrm{~s}, 3 \mathrm{H}), 1.25-1.15(\mathrm{~m}, 1 \mathrm{H}), 1.00-0.81(\mathrm{~m}, 4 \mathrm{H}),{ }^{13} \mathrm{C}$ NMR $(100$ $\left.\mathrm{MHz}, \mathrm{CDCl}_{3}\right): \delta=180.8,143.3,134.3,127.5,122.4,107.8,48.4,40.1,37.6,32.4$, 30.6, 26.1, 25.1, $23.8 \mathrm{ppm}$; The NMR spectrum of $\mathbf{8 b}$ displayed relatively impurities in the aliphatic and aromatic region which due to other inseparable byproducts. 
HRMS (ESI) calcd for $\mathrm{C}_{17} \mathrm{H}_{23} \mathrm{NNaO}[\mathrm{M}+\mathrm{Na}]^{+}$280.1672, found 286.1670. 


\section{Isotope Labeling Experiment}

\section{a) Intramolecular Kinetic Isotope Effect (KIE) Experiment:}

$\left[\mathrm{D}_{1}\right]$-1a was synthesized according the literature procedure. ${ }^{1}$ An oven-dried sealed tube was charged with $\left[\mathrm{D}_{1}\right]-\mathbf{1 a}\left(0.2 \mathrm{mmol}, 1.0\right.$ equiv), $\mathrm{PdCl}_{2}(3.4 \mathrm{mg}, 10 \mathrm{~mol} \%)$, dppf (11.8 mg, $10 \mathrm{~mol} \%$ ), and $\mathrm{K}_{3} \mathrm{PO}_{4} \cdot 3 \mathrm{H}_{2} \mathrm{O}(106 \mathrm{mg}, 0.4 \mathrm{mmol})$. The tube was evacuated and backfilled with nitrogen (three times). Then, a solution of iodocyclohexane 2a ( $0.5 \mathrm{mmol}, 2.5$ equiv) in diglyme $(2.0 \mathrm{~mL})$ was injected by syringe. The tube was then sealed with a Teflon lined cap and placed into a preheated oil bath at $100{ }^{\circ} \mathrm{C}$ with vigorous stirring. After $24 \mathrm{~h}$, the resulting mixture was diluted with EtOAc, and the organic phase was washed successively with $\mathrm{H}_{2} \mathrm{O}$ (three times) and brine (one time) then dried $\left(\mathrm{Na}_{2} \mathrm{SO}_{4}\right)$ and concentrated in vacuo. The residue was purified by column chromatography on silica gel to give the corresponding product. The product was analyzed by ${ }^{1} \mathrm{H}$ NMR (Figure 1).
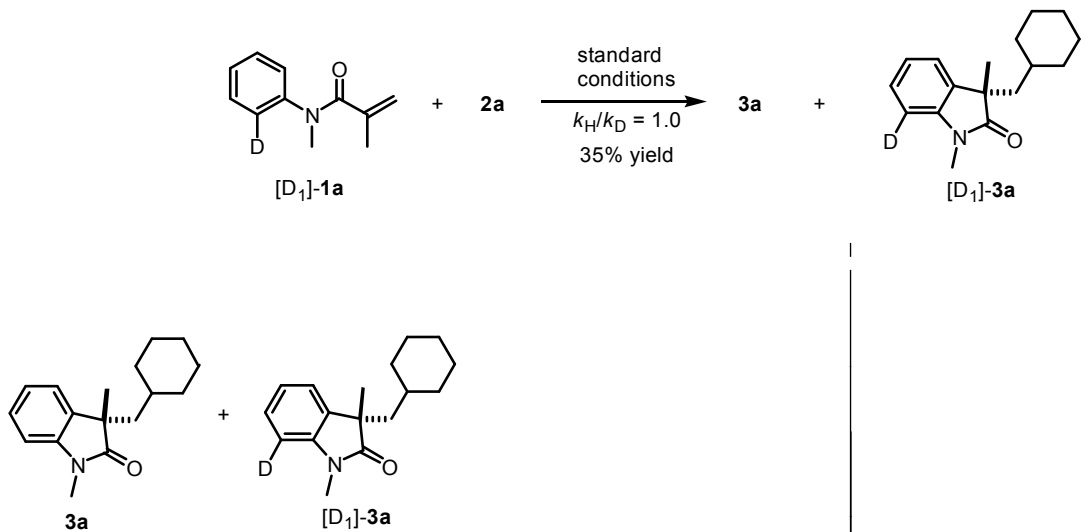

$3 a$
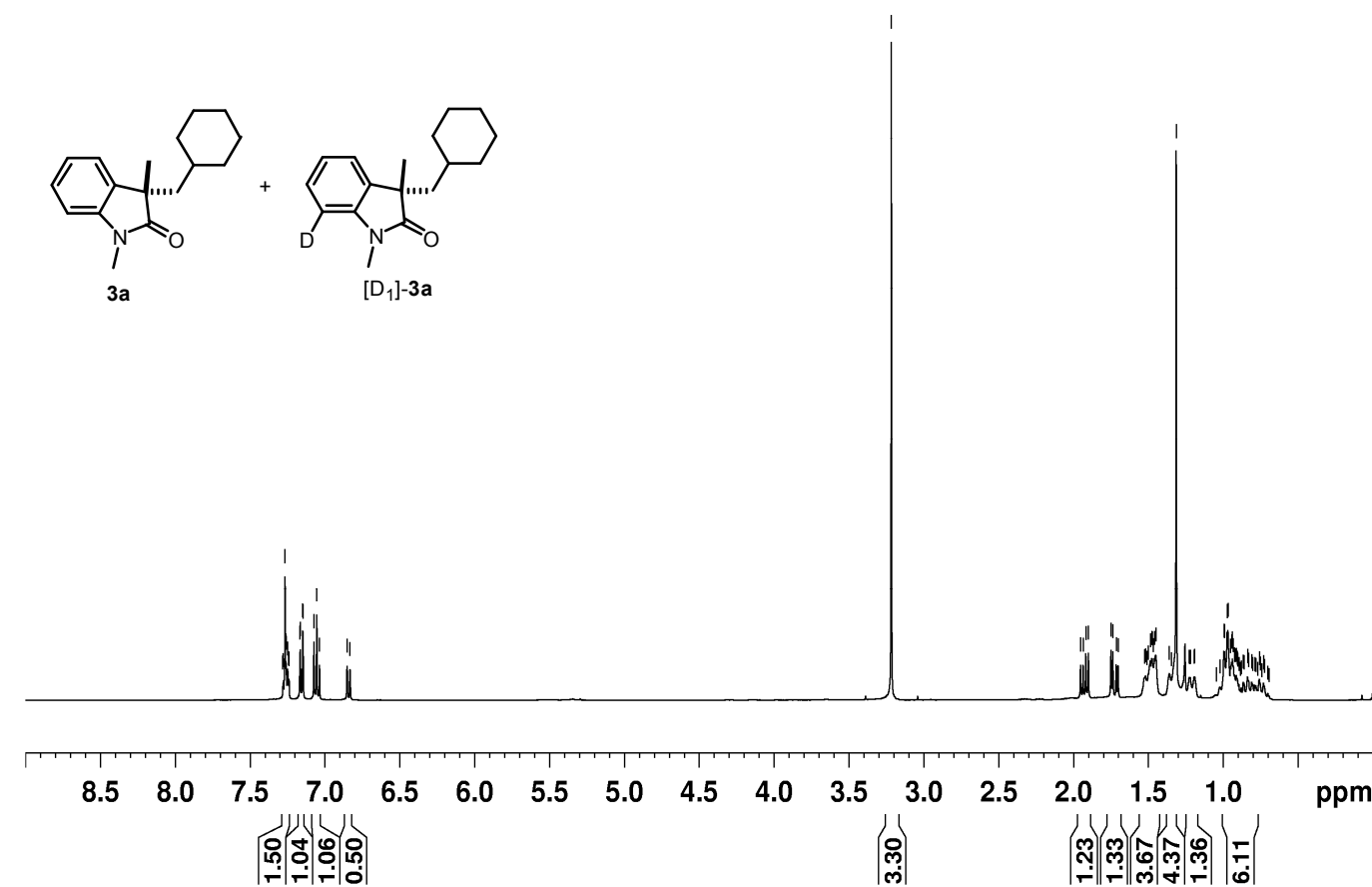

Figure 1. ${ }^{1} \mathrm{H}$ NMR spectra of the mixture of the product $\mathbf{3 a}$ and $\left[\mathrm{D}_{1}\right]-\mathbf{3} \mathbf{a}$. 


\section{b) Intermolecular Kinetic Isotope Effect (KIE) Experiment:}

Aniline (ring- $\mathrm{D}_{5}, 98 \%$, cat. No. DLM-862-0) were purchased from Cambridge Isotope Laboratories. The isotope reagent was used without further purification. $\left[\mathrm{D}_{5}\right]-\mathbf{1 a}$ was synthesized according the literature procedure using $\mathrm{D}_{5}$-aniline as starting material. ${ }^{1}$ An oven-dried sealed tube was charged with $\left[\mathrm{D}_{5}\right]-1 \mathrm{a}(0.1 \mathrm{mmol}), \mathbf{1 a}(0.1 \mathrm{mmol})$, $\mathrm{PdCl}_{2}$ (3.4 mg, $10 \mathrm{~mol} \%$ ), dppf (11.8 mg, $10 \mathrm{~mol} \%$ ), and $\mathrm{K}_{3} \mathrm{PO}_{4} \cdot 3 \mathrm{H}_{2} \mathrm{O}(106 \mathrm{mg}, 0.4$ mmol). The tube was evacuated and backfilled with nitrogen (three times). Then, a solution of iodocyclohexane $\mathbf{2 a}(0.5 \mathrm{mmol}, 2.5$ equiv) in diglyme $(2.0 \mathrm{~mL})$ was injected by syringe. The tube was then sealed with a Teflon lined cap and placed into a preheated oil bath at $100{ }^{\circ} \mathrm{C}$ with vigorous stirring. After $24 \mathrm{~h}$, the resulting mixture was diluted with EtOAc, and the organic phase was washed successively with $\mathrm{H}_{2} \mathrm{O}$ (three times) and brine (one time) then dried $\left(\mathrm{Na}_{2} \mathrm{SO}_{4}\right)$ and concentrated in vacuo. The residue was purified by column chromatography on silica gel to give the corresponding product. The product was analyzed by ${ }^{1} \mathrm{H}$ NMR (Figure 2).
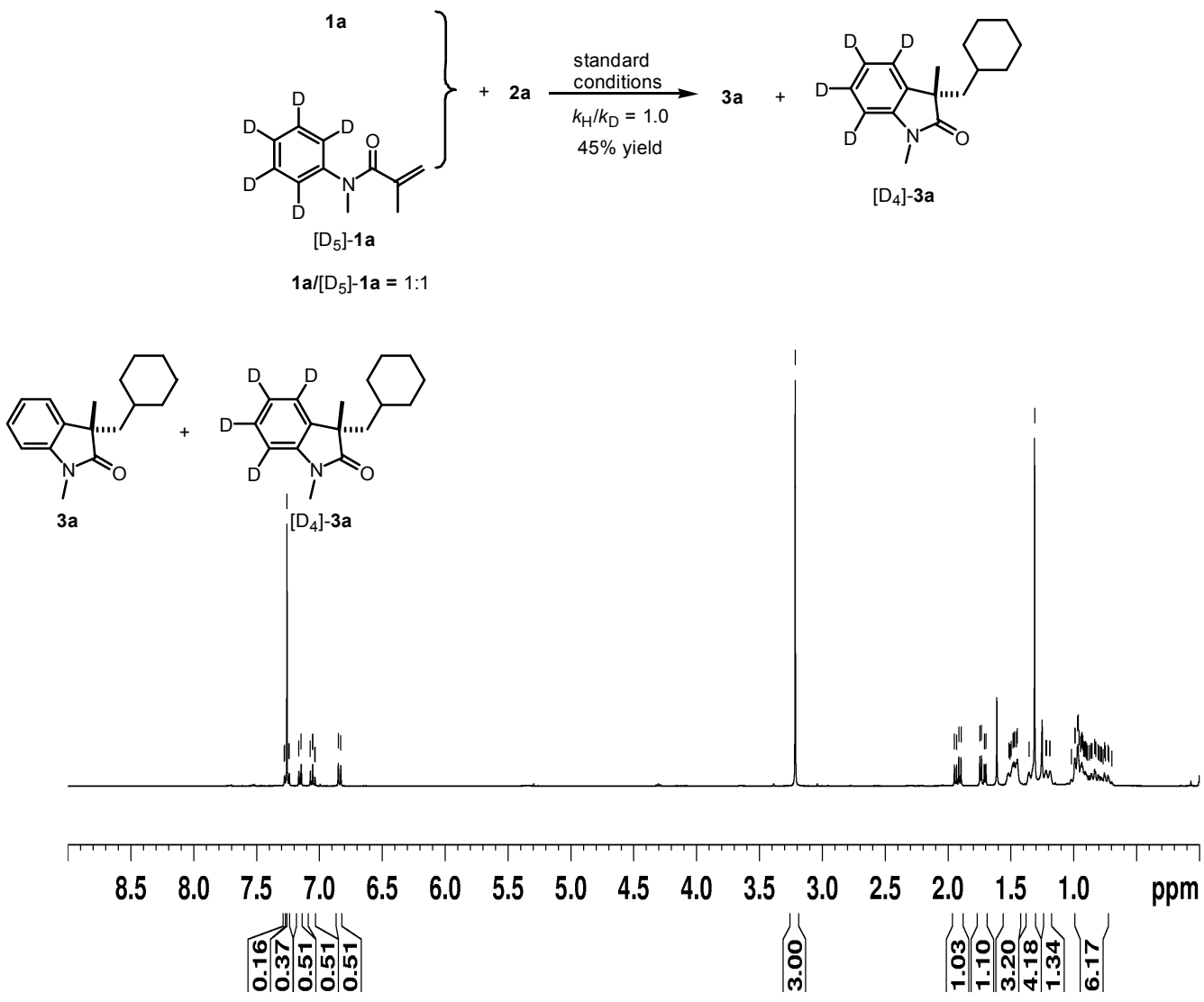

Figure 2. ${ }^{1} \mathrm{H}$ NMR spectra of the mixture of the product $\mathbf{3 a}$ and $\left[\mathrm{D}_{4}\right]-\mathbf{3 a}$. 


\section{Proposed Mechanism}

According to the above control experiments, a possible mechanism is proposed in Figure 3. Fristly, carbon-centered radical I is formed by single-electron oxidative addition of the iodocyclohexane in the presence of $\operatorname{Pd}(0)$. Subsequently, the radical $\mathbf{I}$ adds to the $\mathrm{C}=\mathrm{C}$ bond of acrylamide to give alkyl radical II, which undergoes an intramolecular cyclization to generate radical intermediate III. Finally, a Pd(I)-mediated single-electron oxidation of radical III into the corresponding carbocation followed by the loss of $\mathrm{H}^{+}$, leads to the desired product 3a and regenerates $\operatorname{Pd}(0)$.

Figure 3. Proposed Mechanism

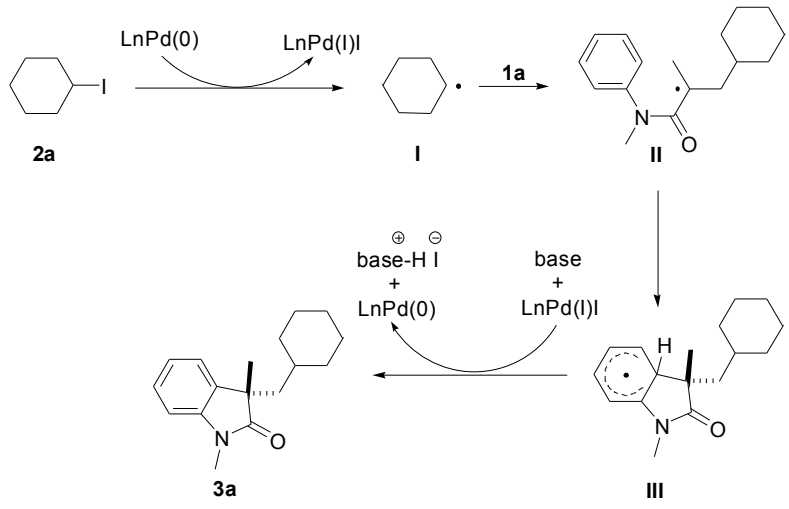




\section{References}

(1) (a) Wu, T.; Mu, X.; Liu, G. Angew. Chem. Int. Ed. 2011, 50, 12578. (b) Mu, X.;

Wu, T.; Wang, H.-Y.; Guo, Y.-L.; Liu, G. J. Am. Chem. Soc. 2012, 134, 878. (c)

Fabry, D. C.; Stodulski, M.; Hoerner, S.; Gulder, T. Chem. Eur. J. 2012, 18, 10834. (d)

McMahon, C. M.; Alexanian, E. J. Angew. Chem., Int. Ed. 2014, 53, 5974. 


\section{${ }^{1}$ H NMR and ${ }^{13} \mathrm{C}$ NMR Spectra of Products 3}

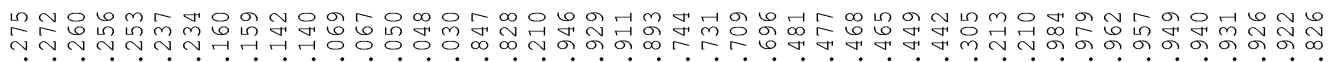

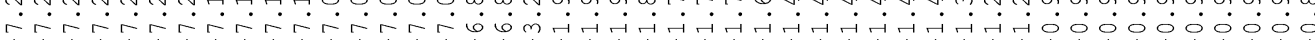

(1)
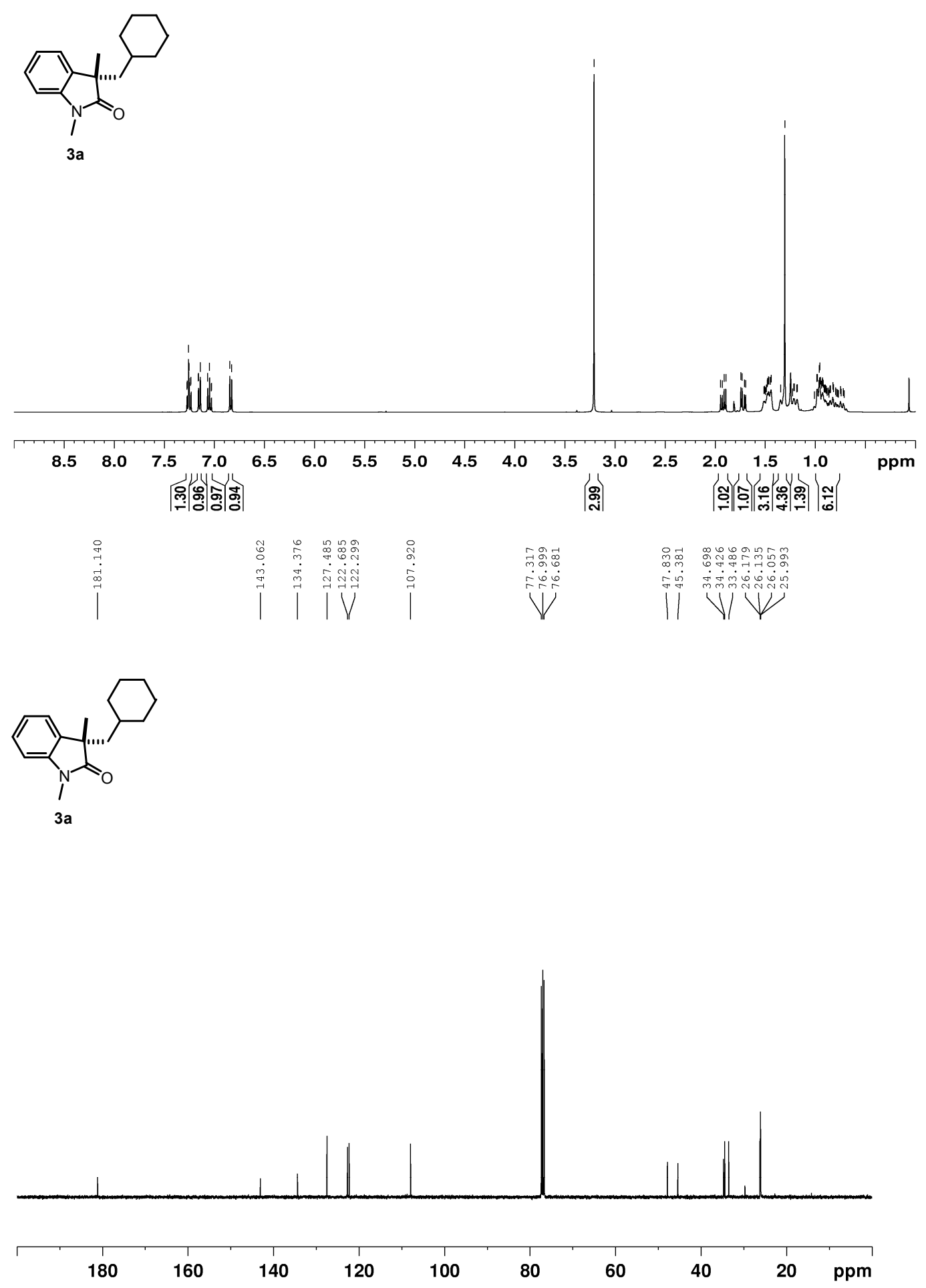

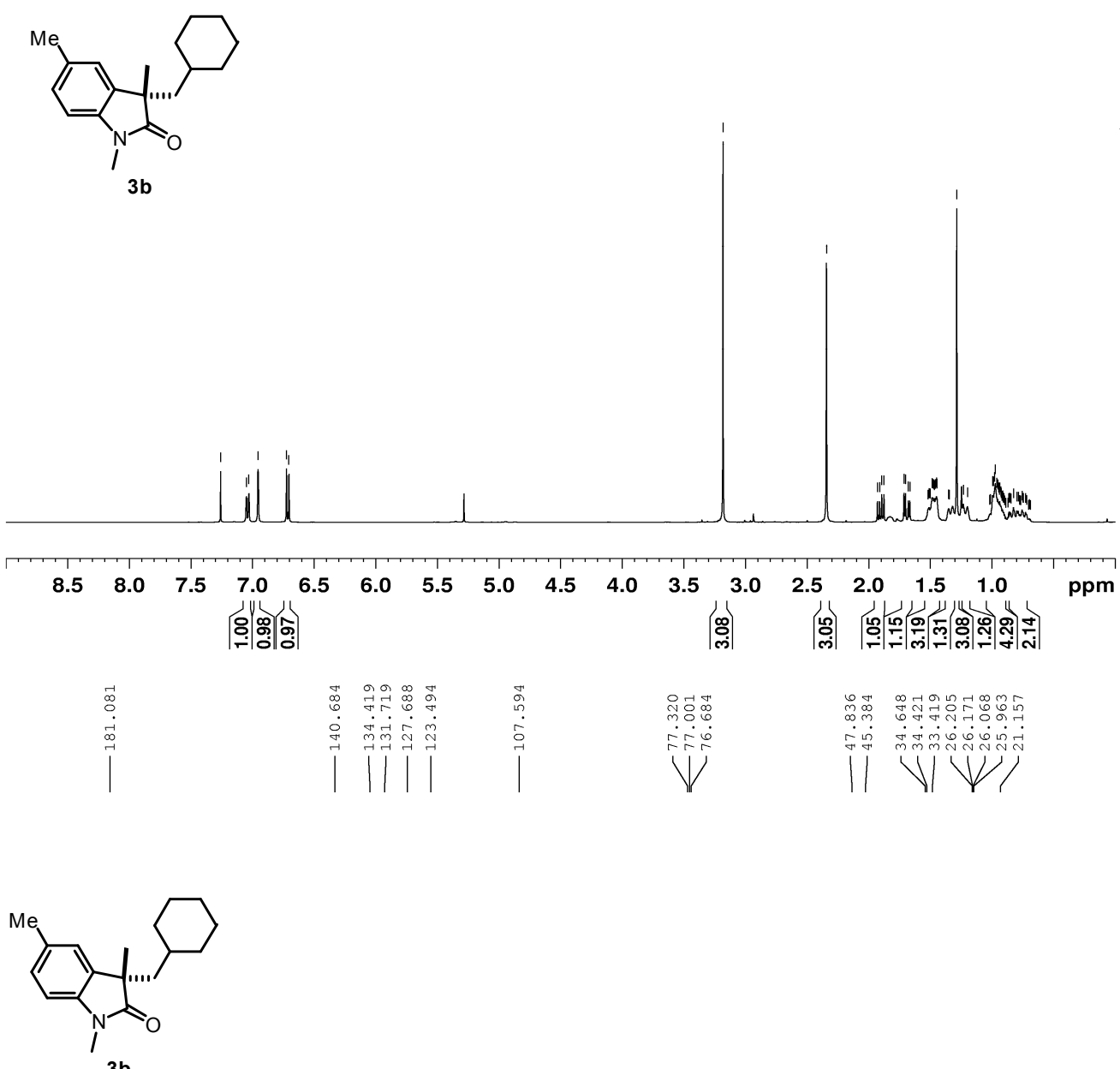

$3 b$

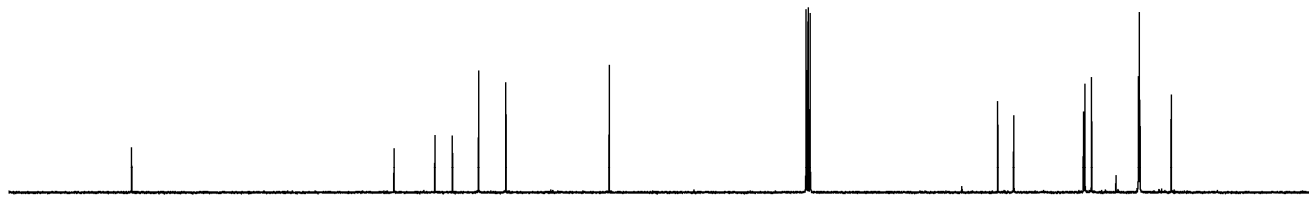

\begin{tabular}{|c|c|c|c|c|c|c|c|c|}
\hline 100 & 10 & & 11 & 11 & 1 & 1 & 11 & \\
\hline 180 & 160 & 140 & 120 & 100 & 80 & 60 & 40 & 20 \\
\hline
\end{tabular}


<smiles>CCCCCN1C(=O)C(C)(CC2CCCCC2)c2cc(OC)ccc21</smiles>

$3 c$
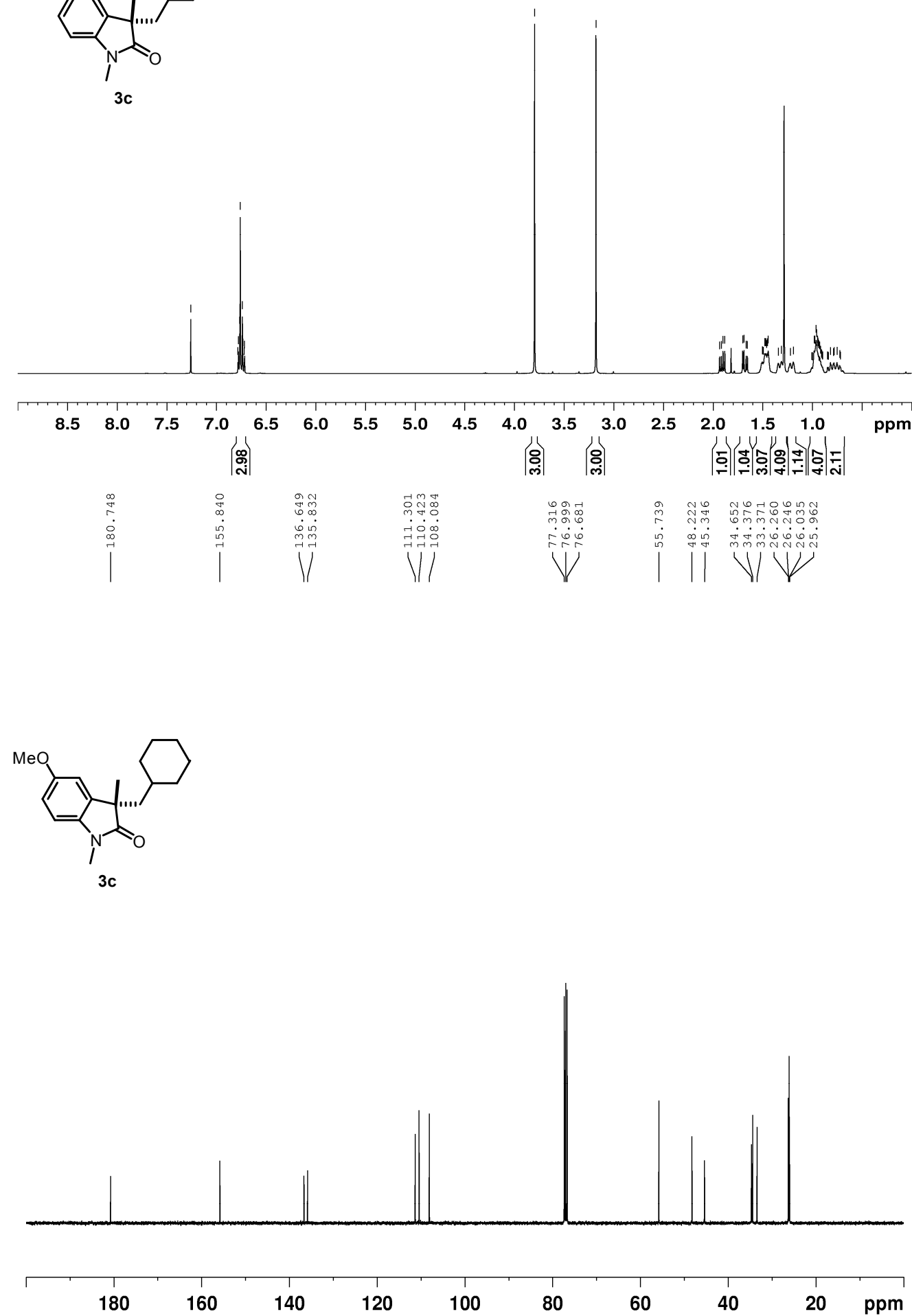
<smiles>CN1C(=O)[C@](C)(CC2CCCCC2)c2cc(C(F)(F)F)ccc21</smiles>

3d
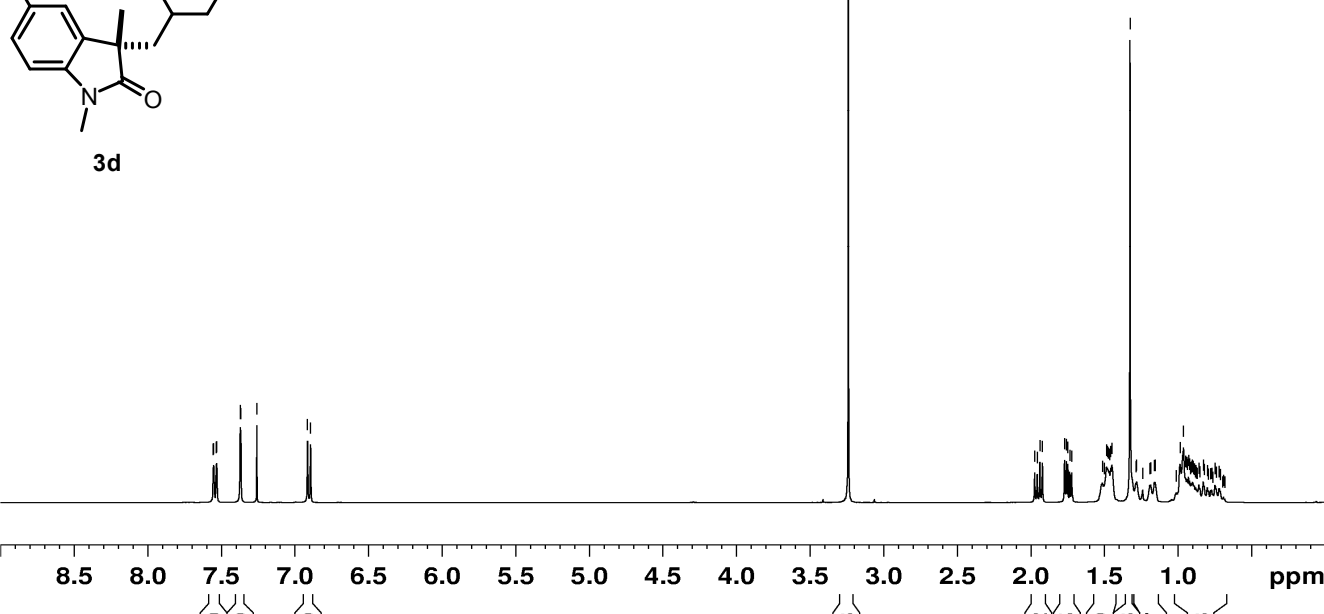
잉영

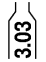

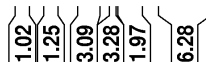
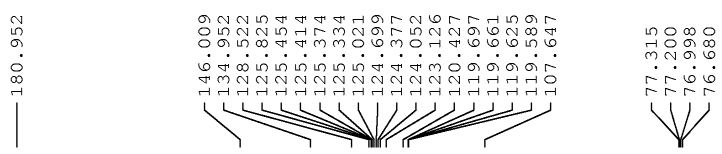

$\underset{m}{m} \begin{array}{ll}\infty \\ \text { m }\end{array}$

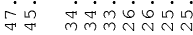

$11 \int^{n}$<smiles>CN1C(=O)[C@](C)(CC2CCCCC2)c2cc(C(F)(F)F)ccc21</smiles>

3d
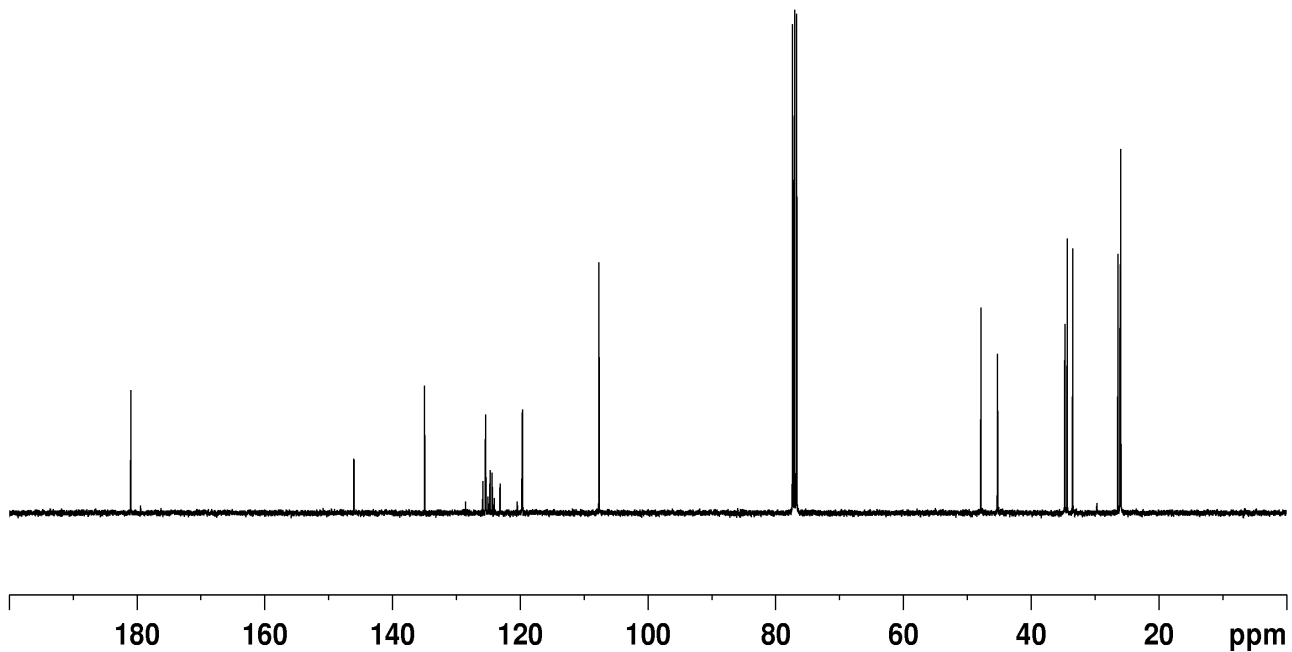

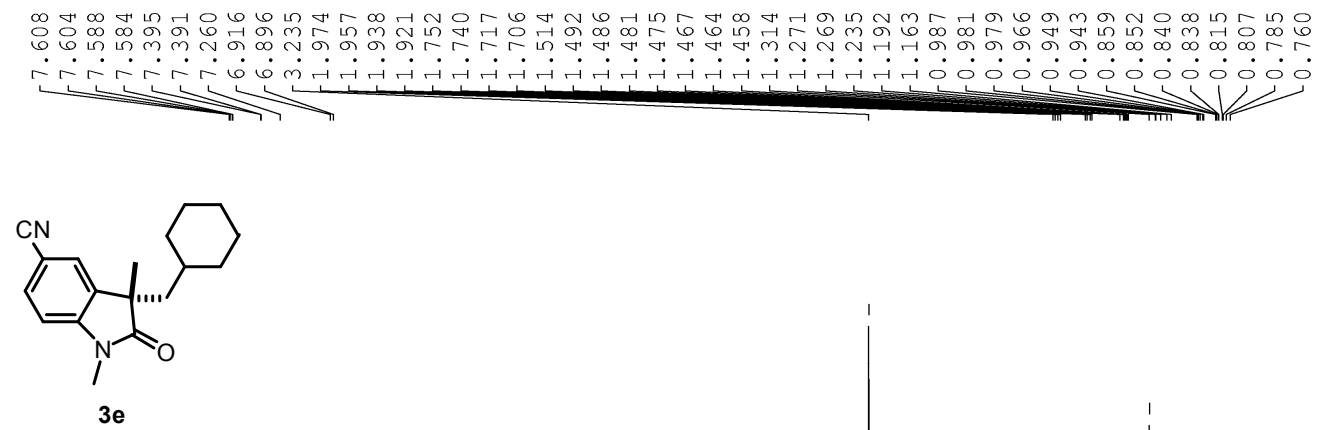

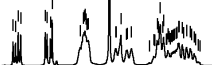
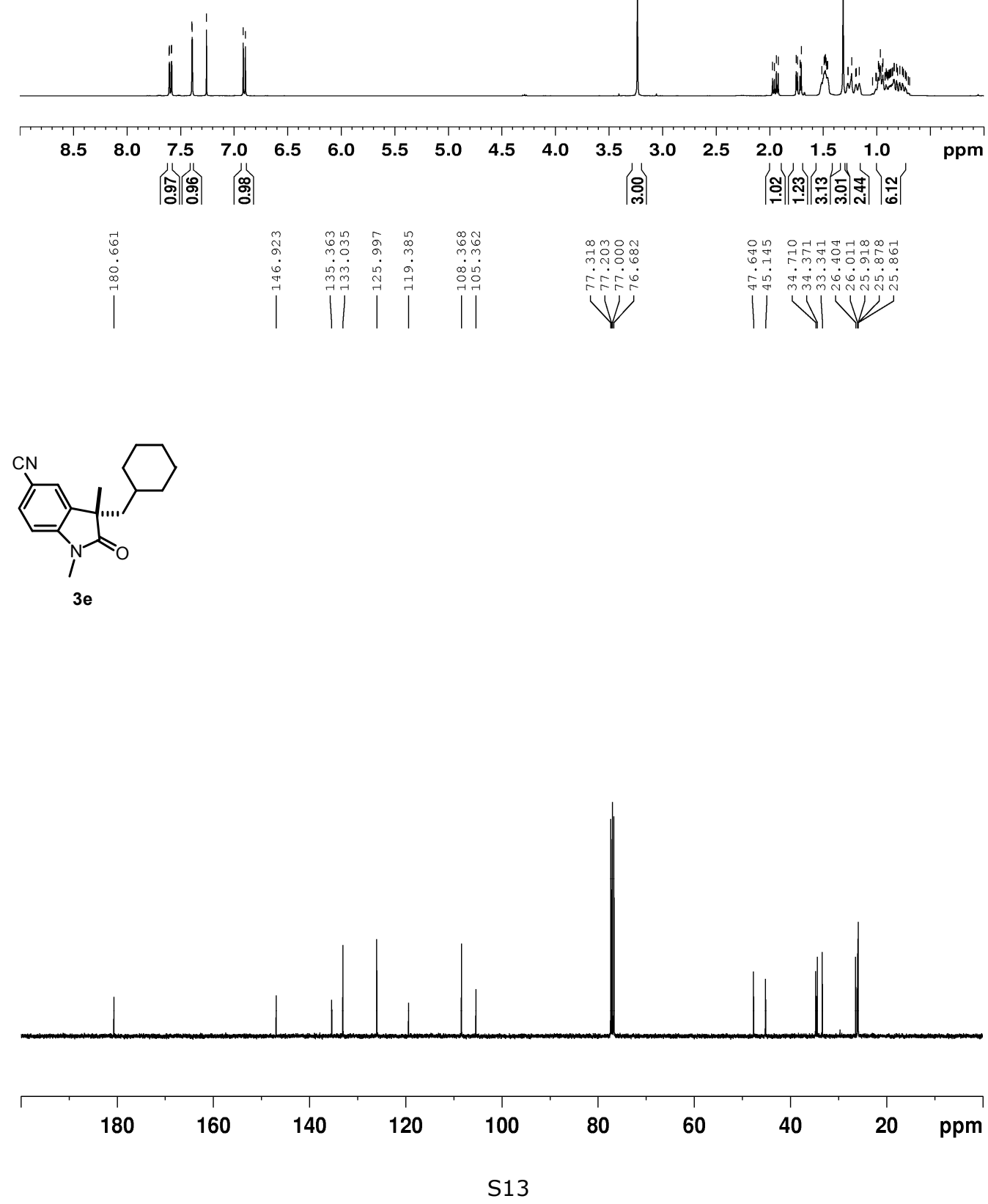

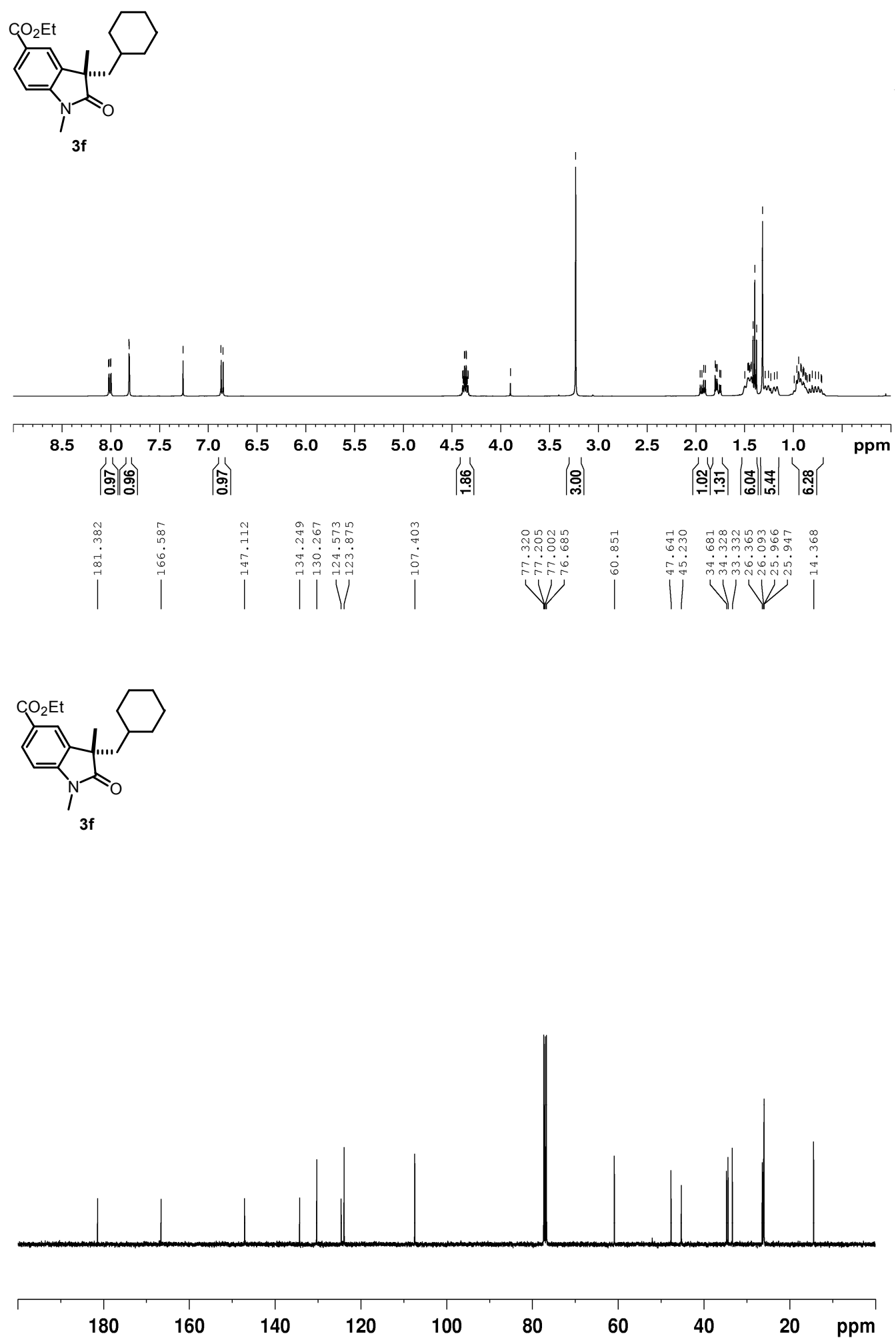
(n)<smiles>CN1C(=O)C(C)(CC2CCCCC2)c2cc(Cl)ccc21</smiles>
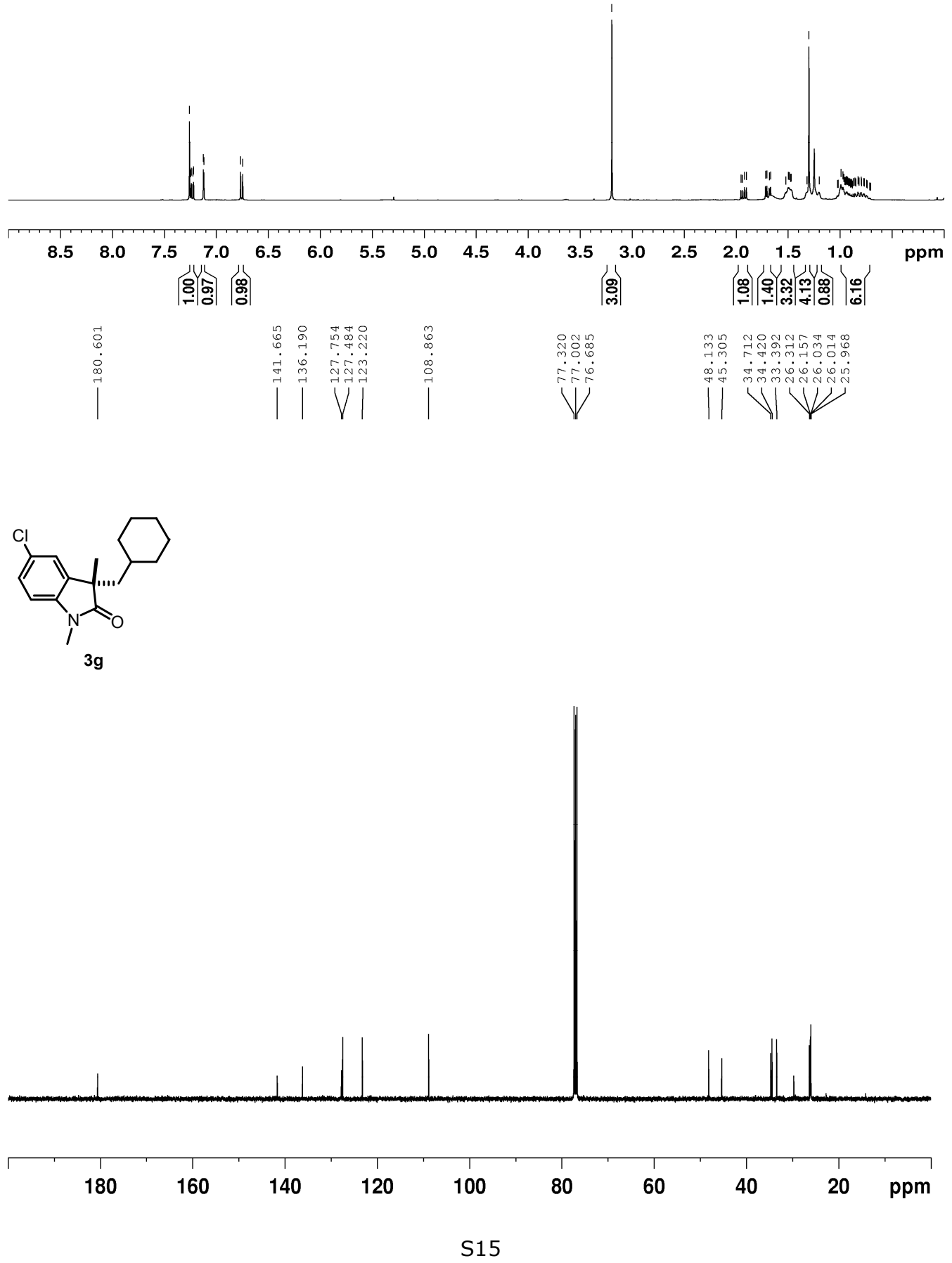
$\longrightarrow m$<smiles>Cc1cccc2c1N(C)C(=O)C2(C)CC1CCCCC1</smiles>

$3 \mathbf{i}$

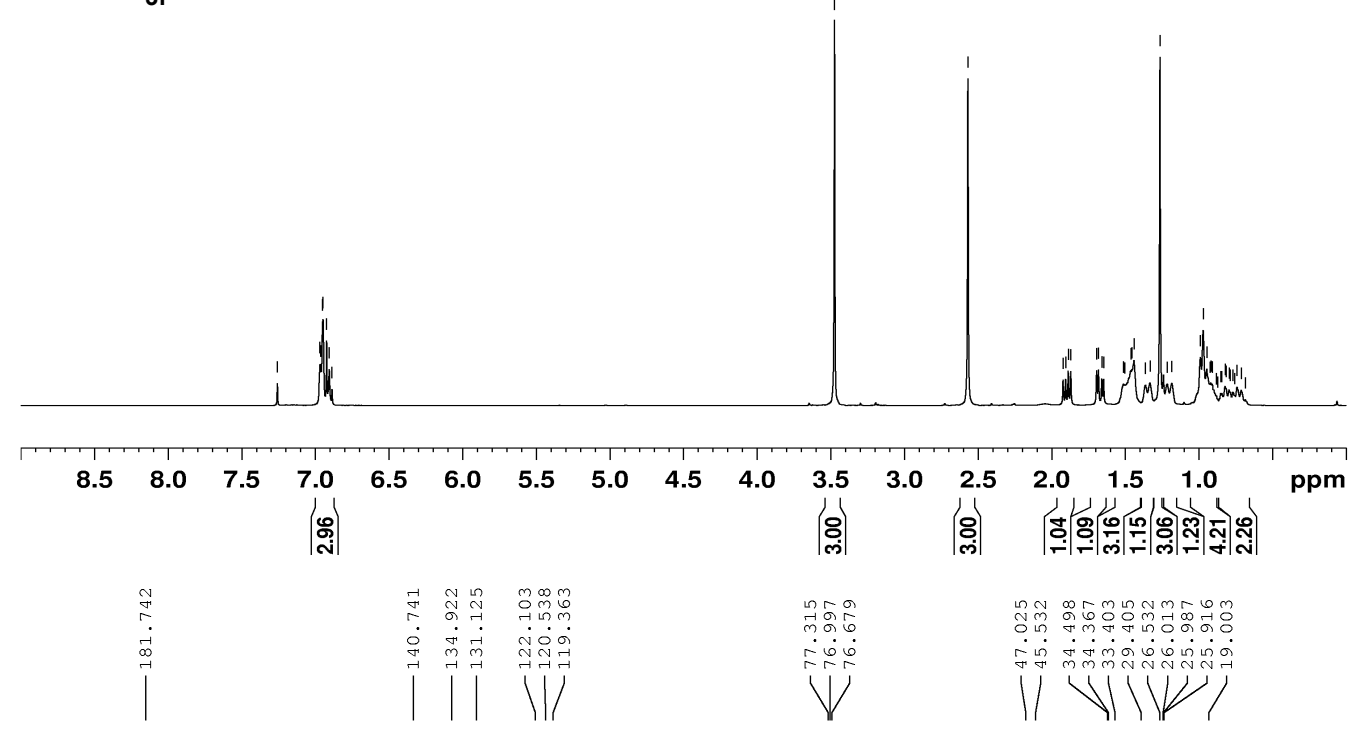<smiles>Cc1cccc2c1N(C)C(=O)[C@]2(C)CC1CCCCC1</smiles>

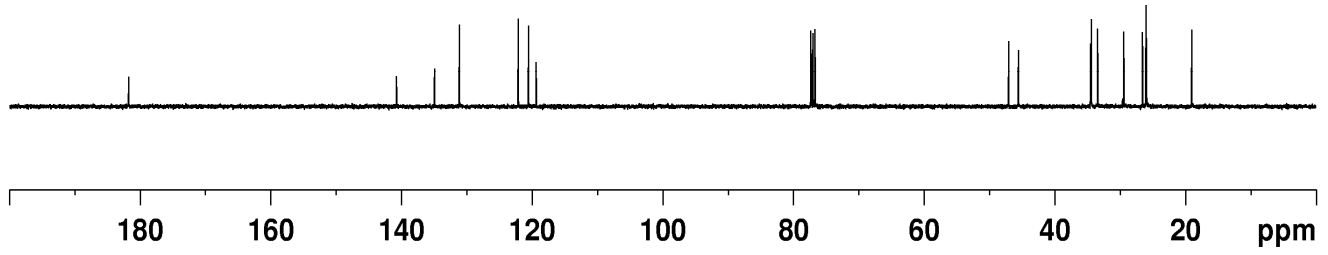




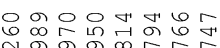

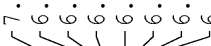

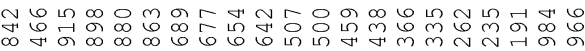

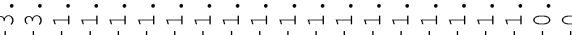

(n)

MeO
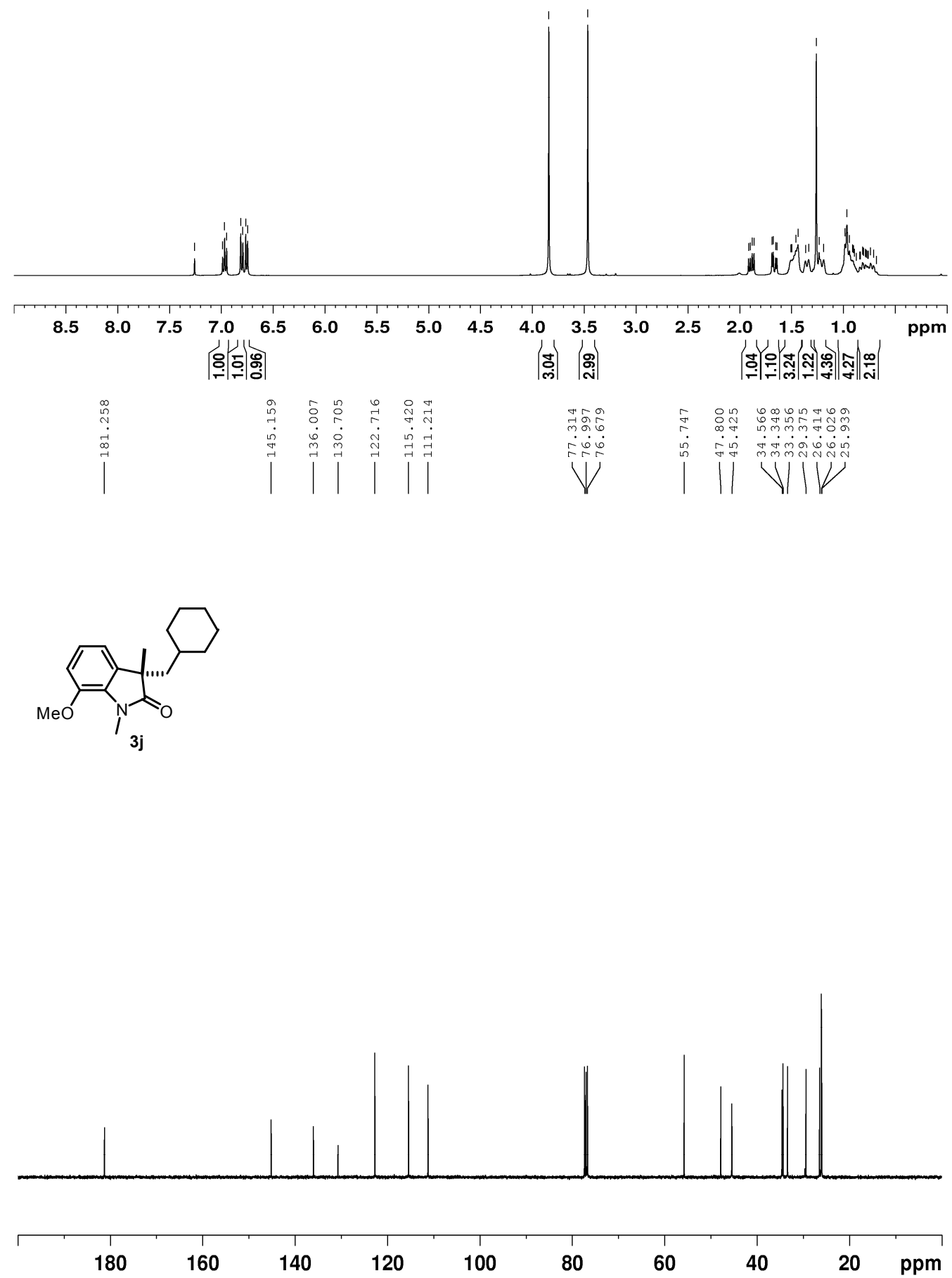


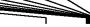<smiles>CN1c2c(F)cccc2C(=O)C1(C)CC1CCCCC1</smiles>

3k
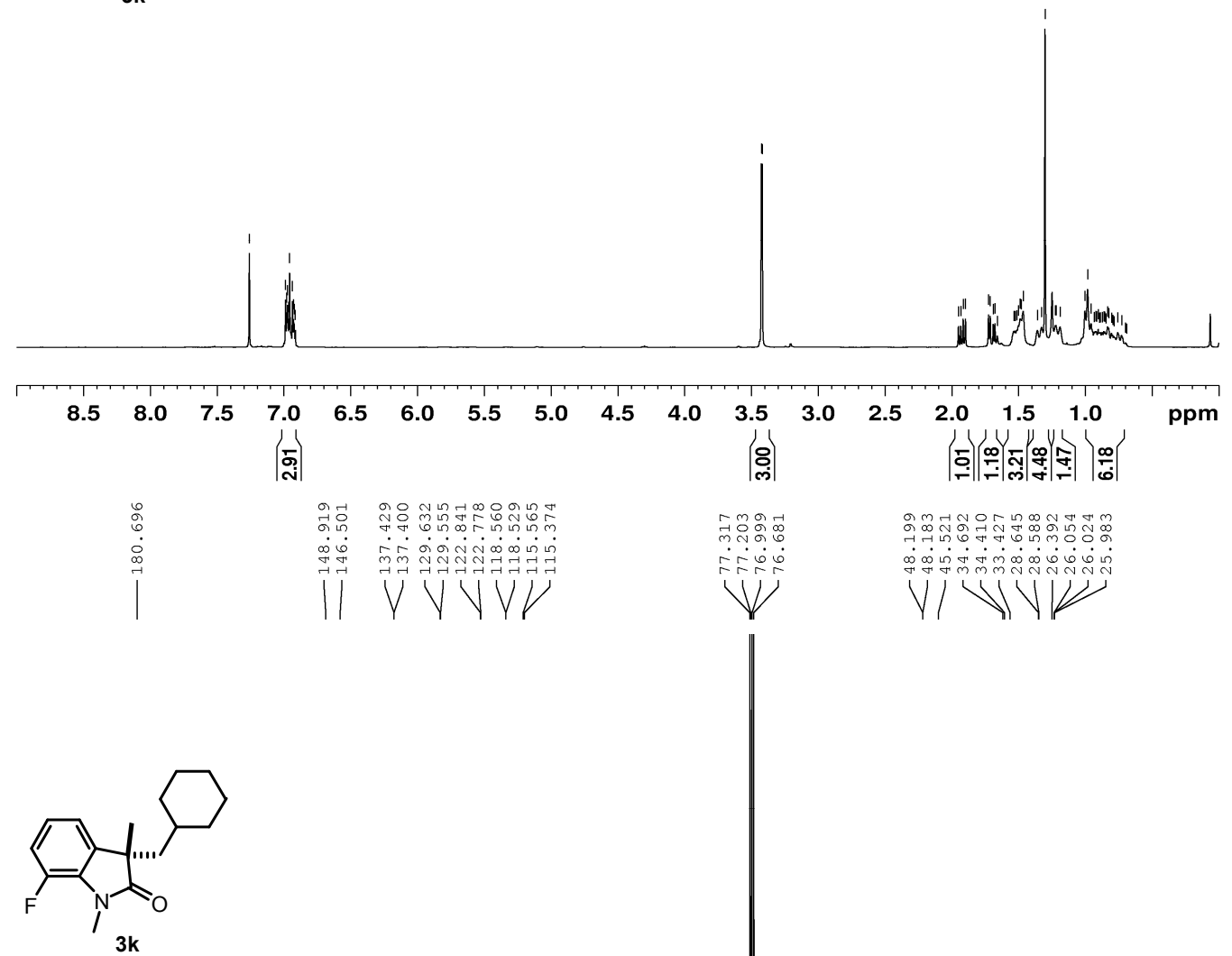

$\mid$

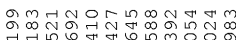
क्ष VIVIN$$
\text { k }
$$ 
<smiles>CN1C(=O)C(C)(CC2CCCCC2)c2cccc(-c3ccccc3)c21</smiles>
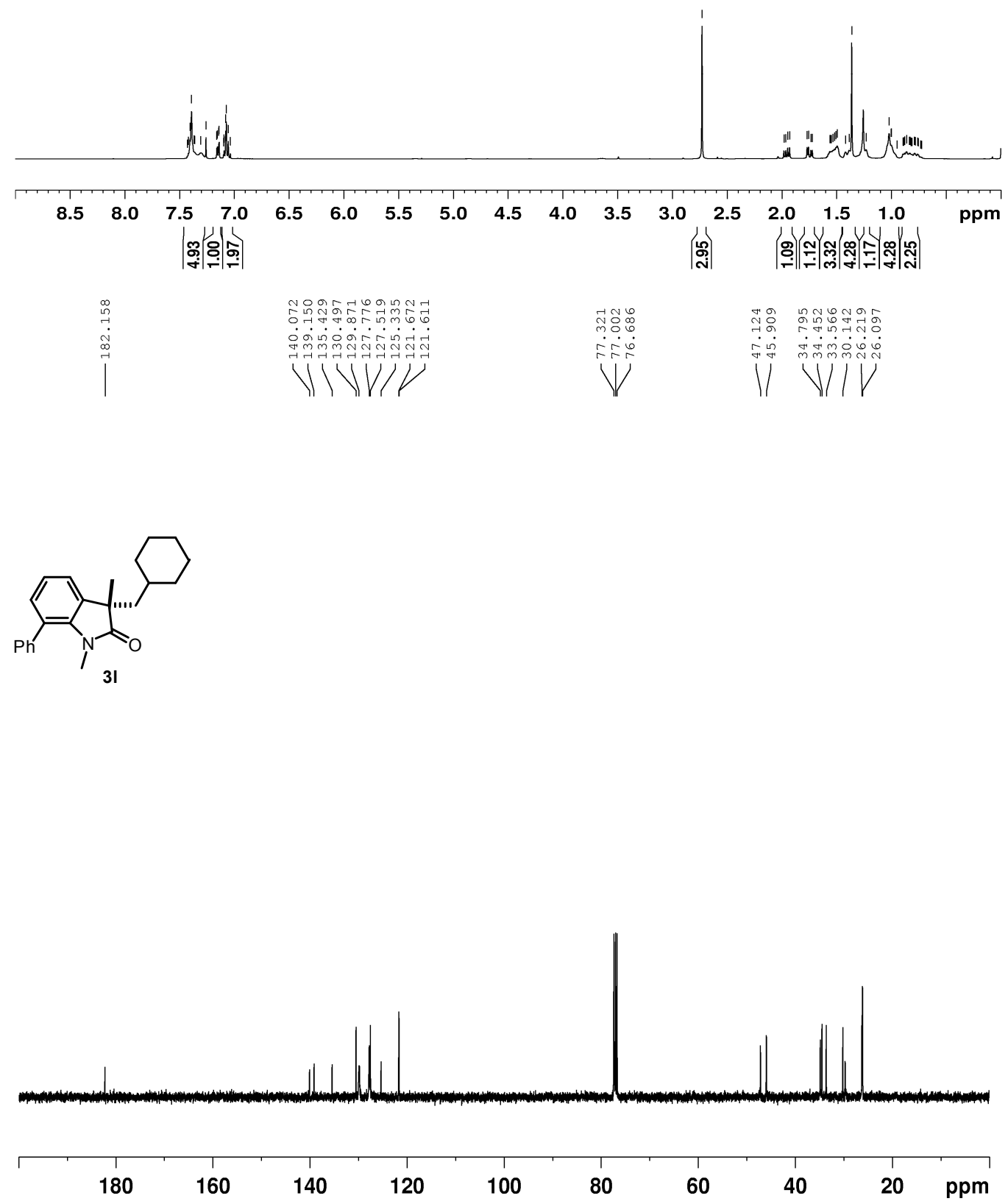
-<smiles>Cc1ccc2c(c1C)N(C)C(=O)[C@]2(C)CC1CCCCC1</smiles>
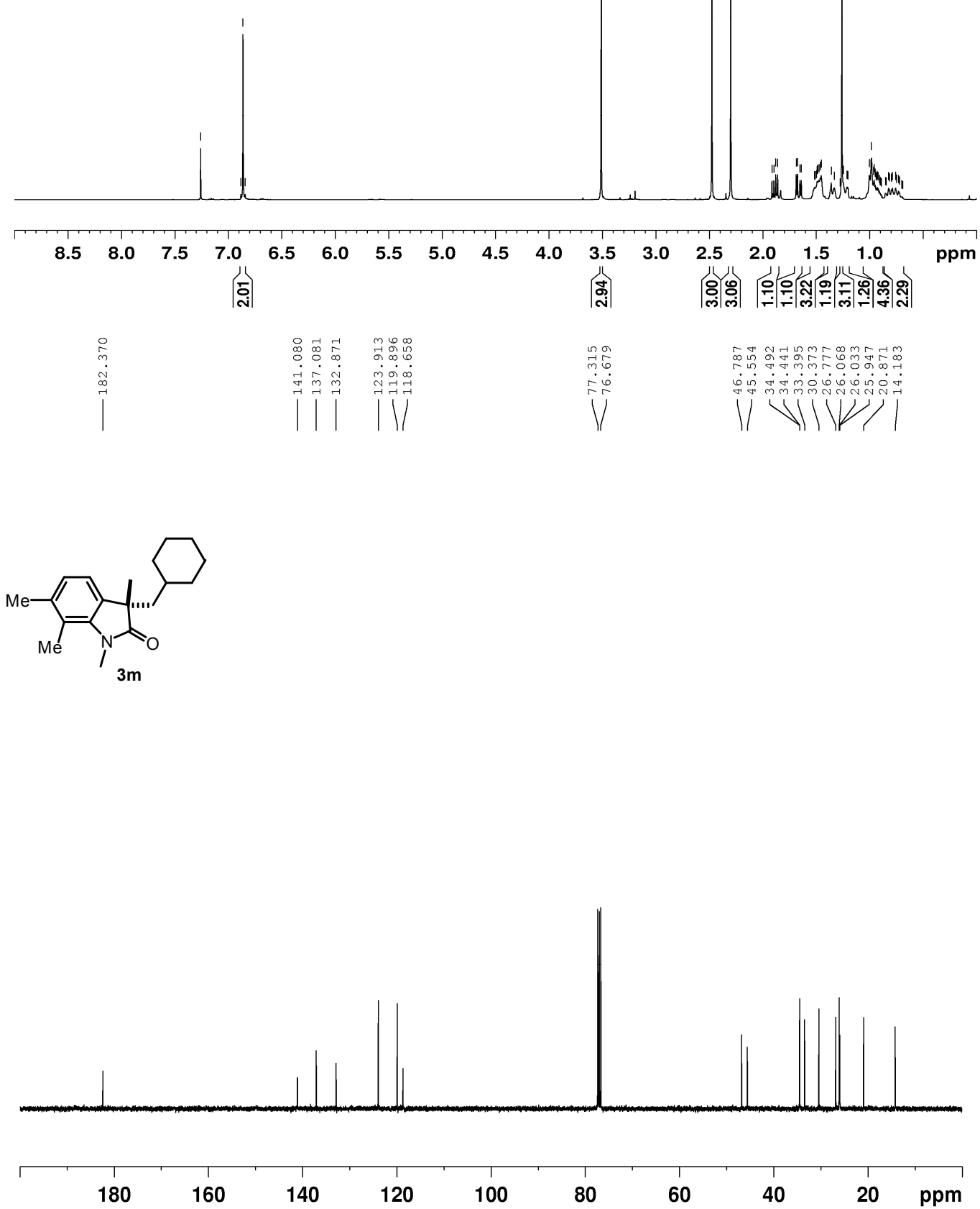


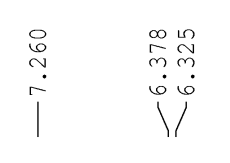

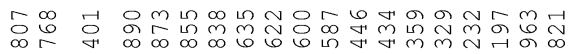

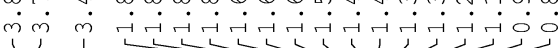
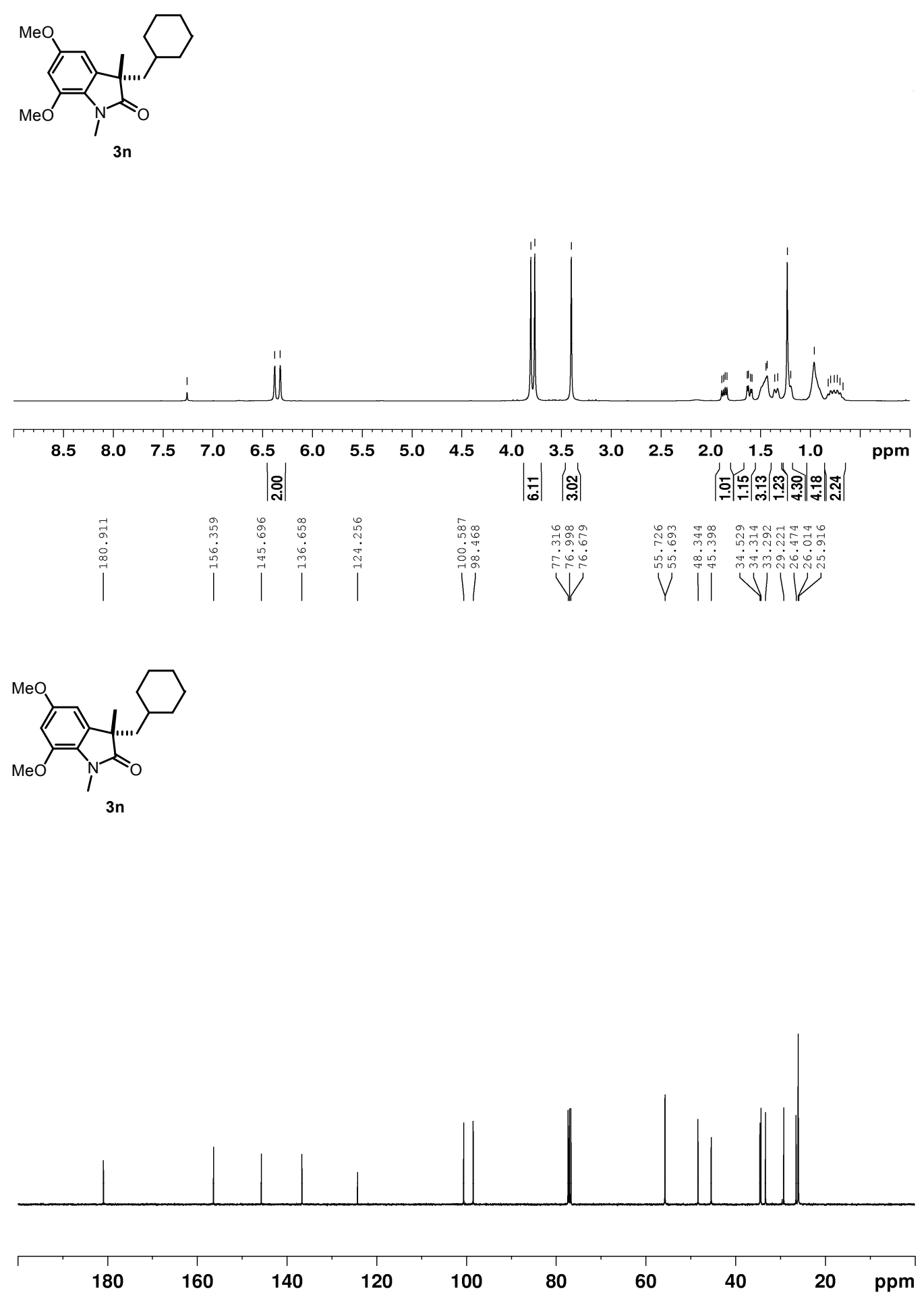


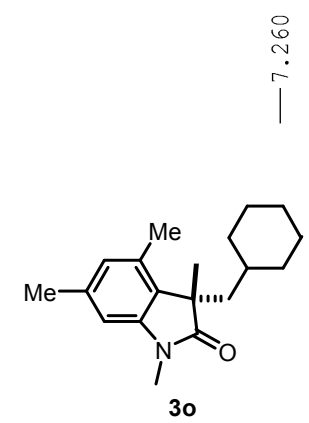

$$
\text { 部 }
$$

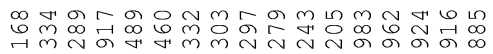

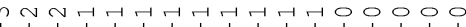
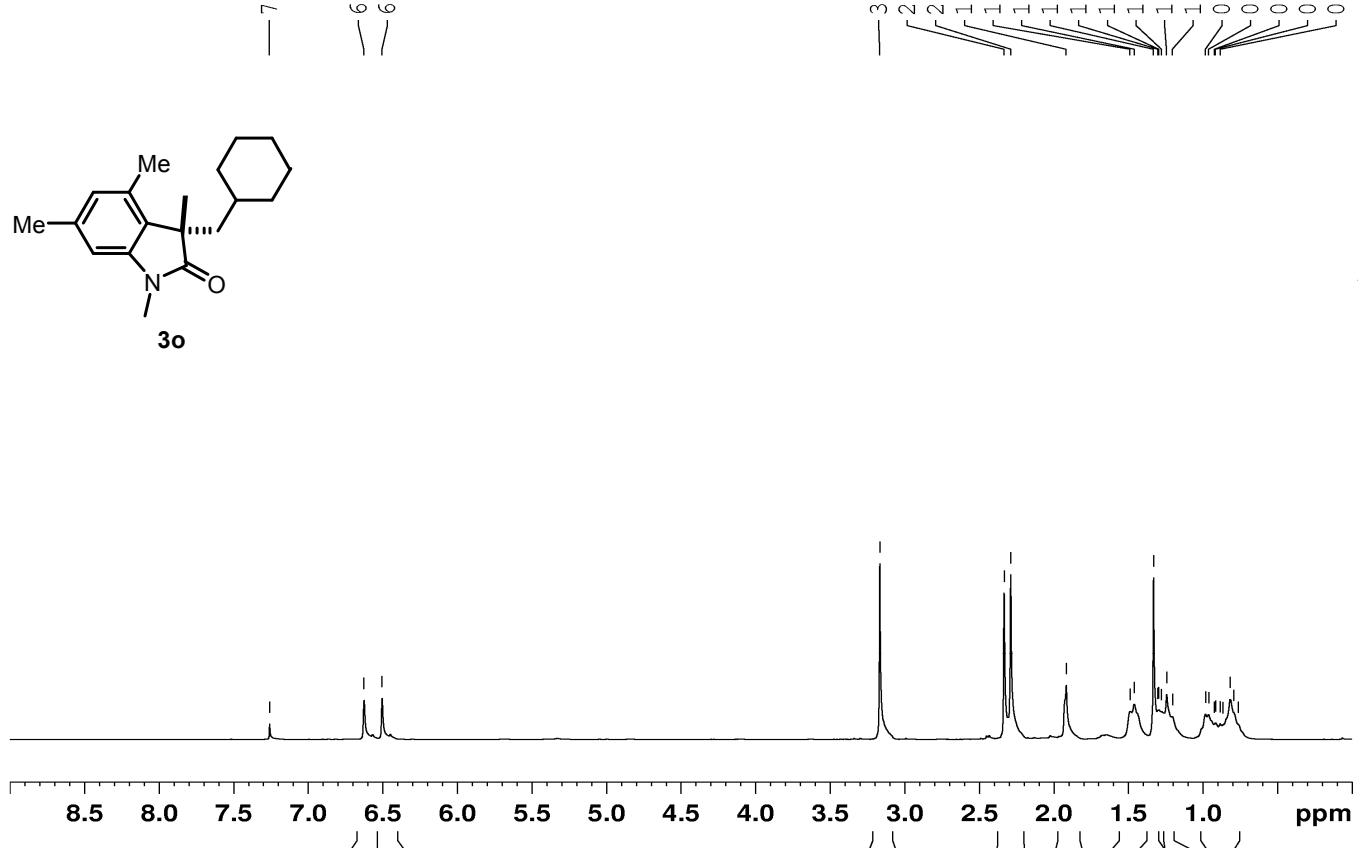
:
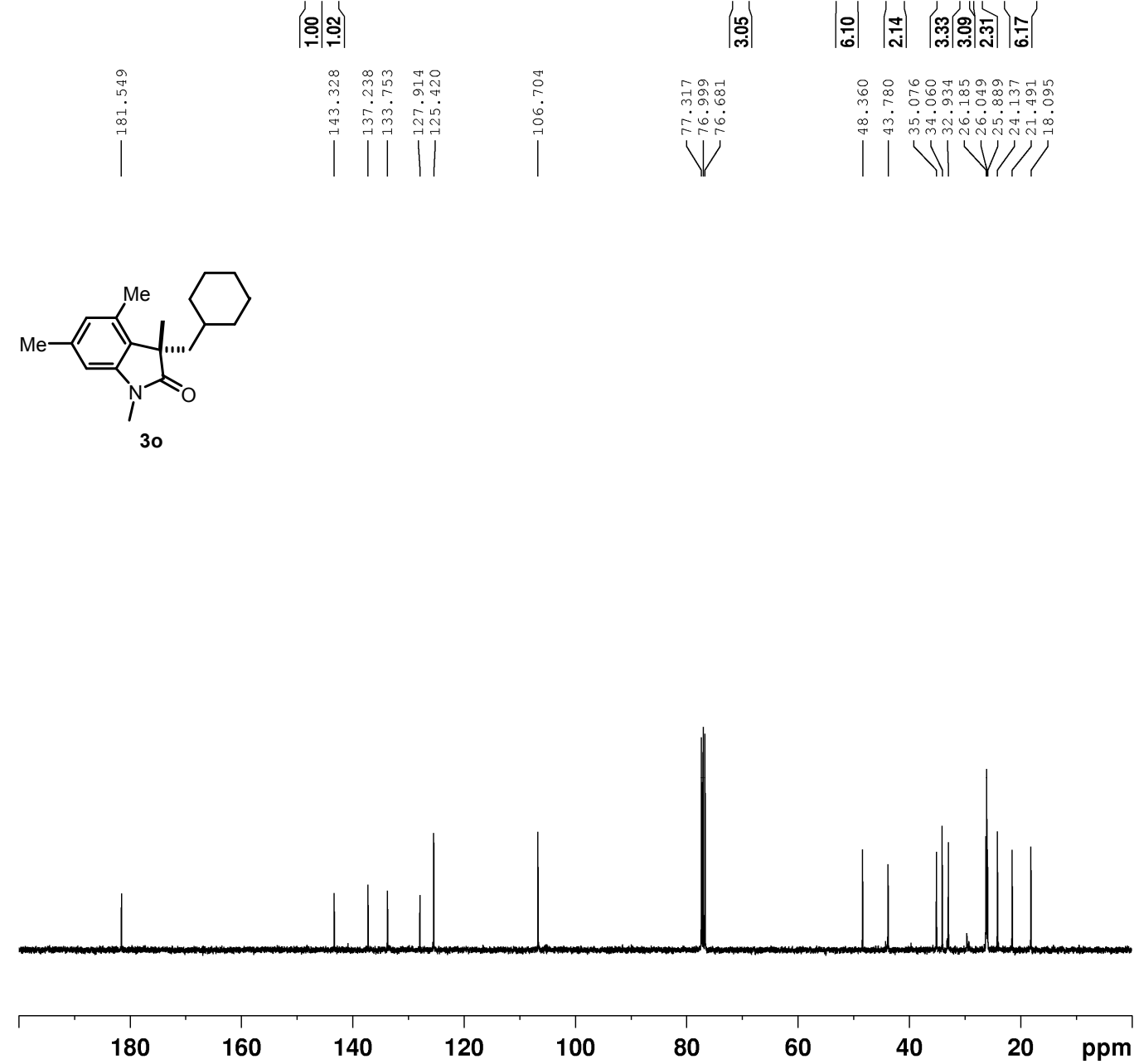
<smiles>CC1(CC2CCCCC2)C(=O)N(Cc2ccccc2)c2ccccc21</smiles>
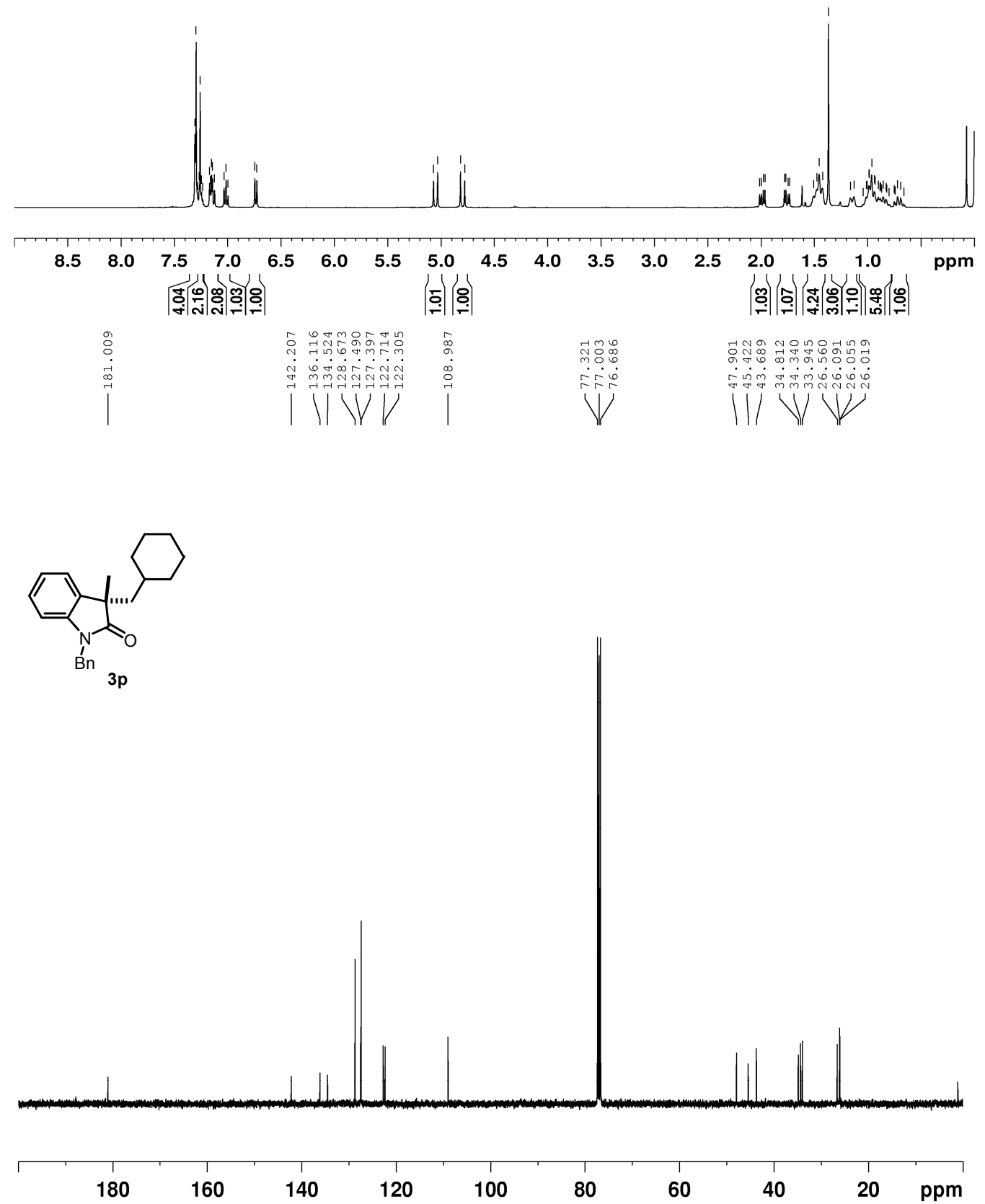


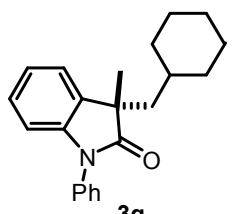

$3 q$
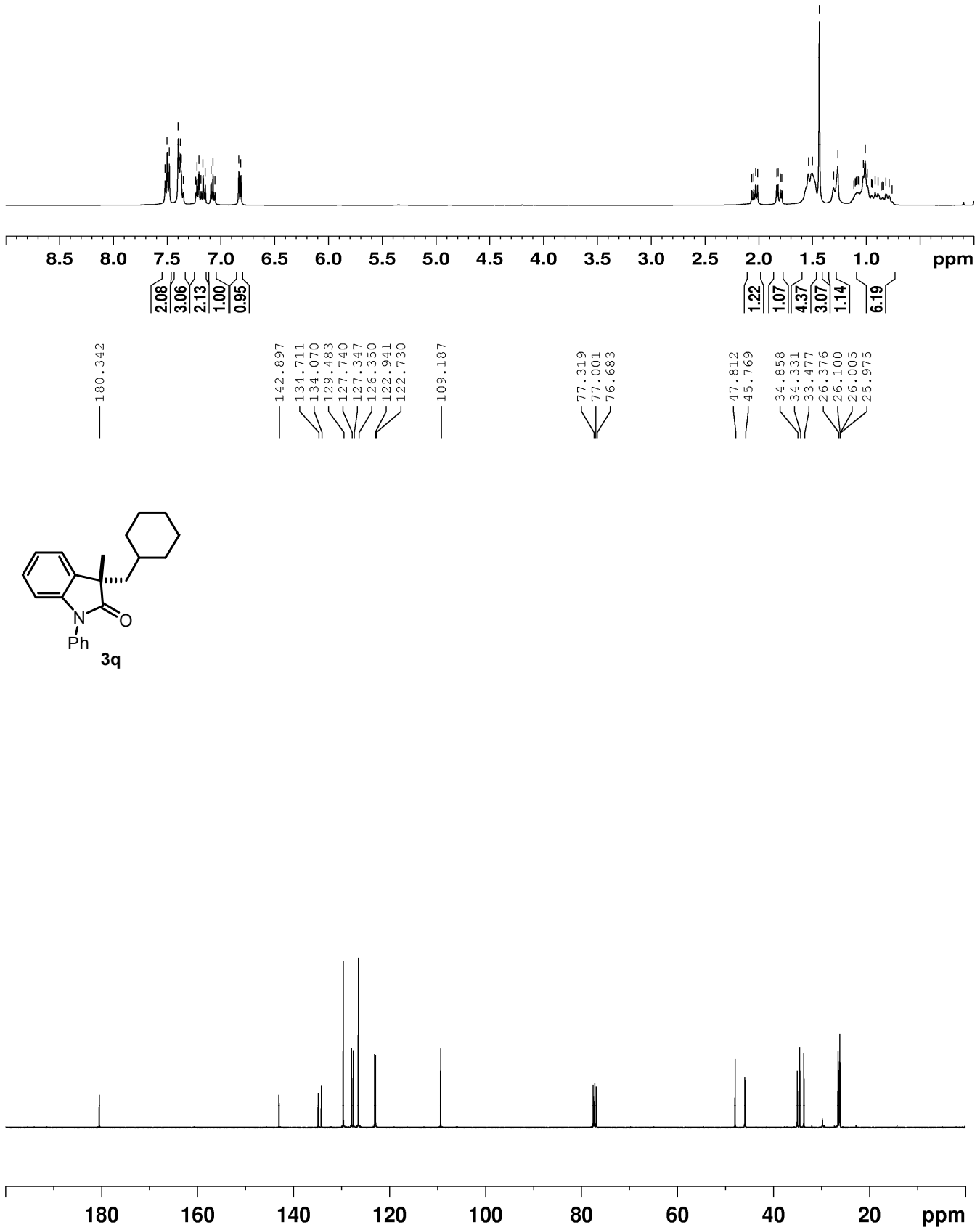

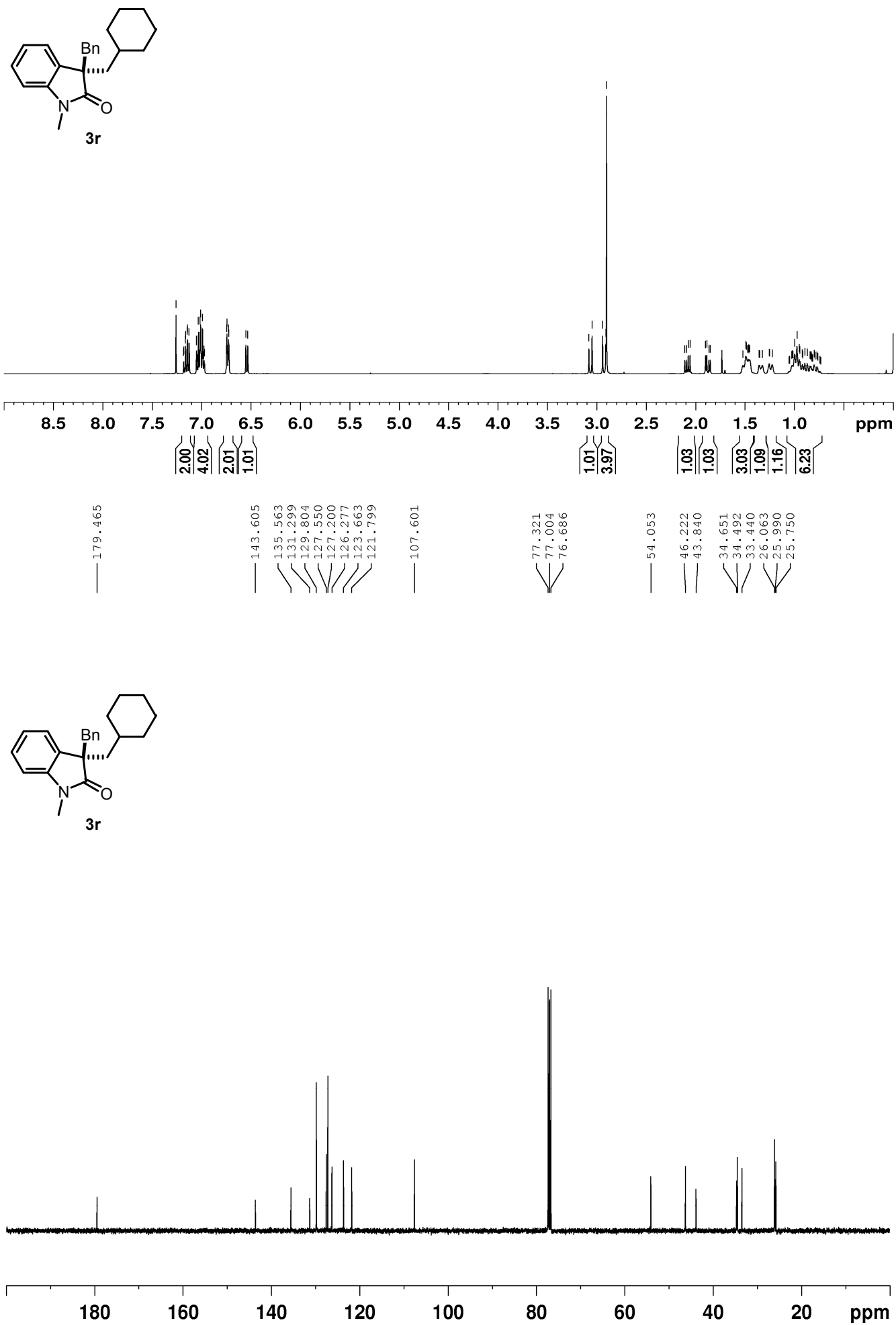

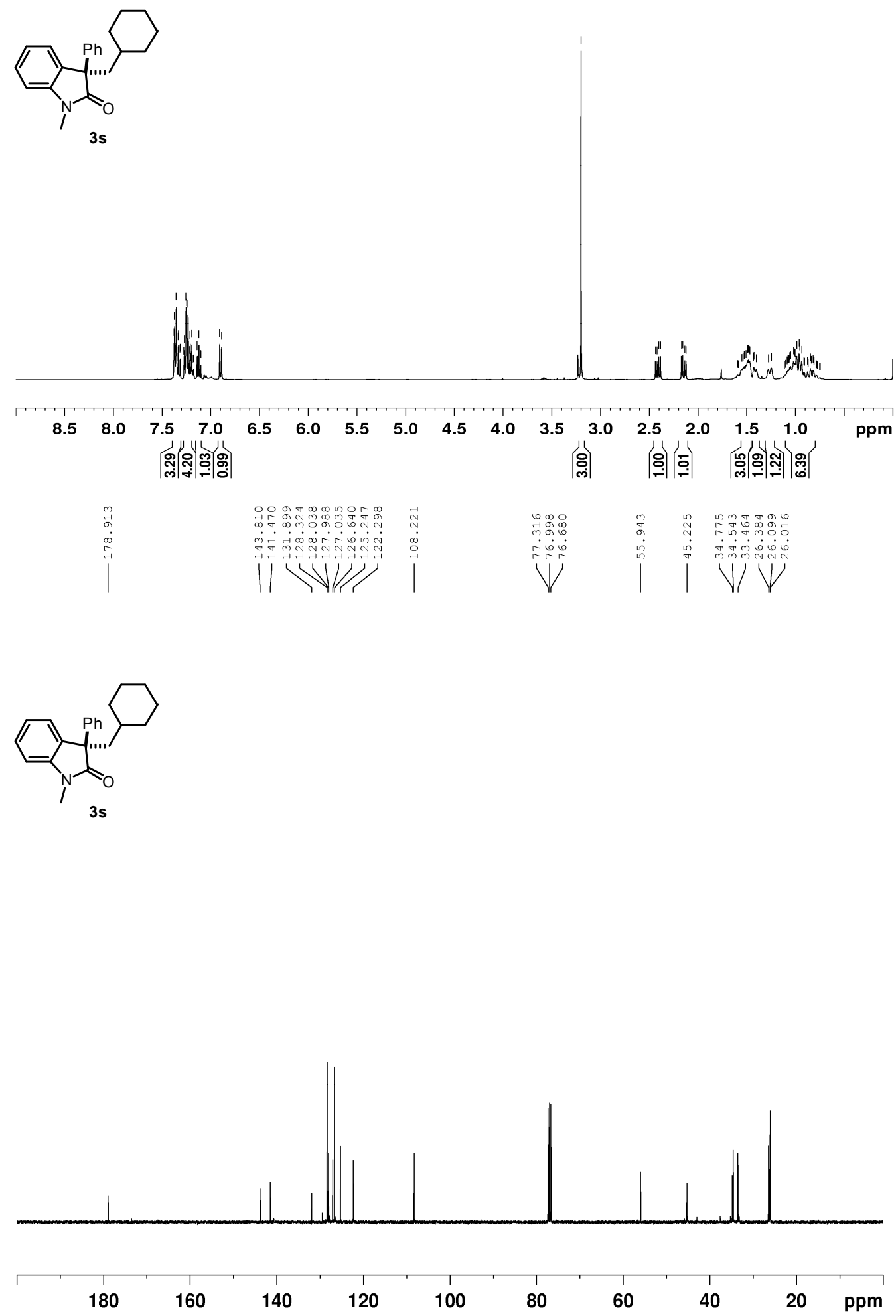
कात?<smiles>Cc1ccc2c(c1)[C@](CO)(CC1CCCCC1)C(=O)N2C</smiles>
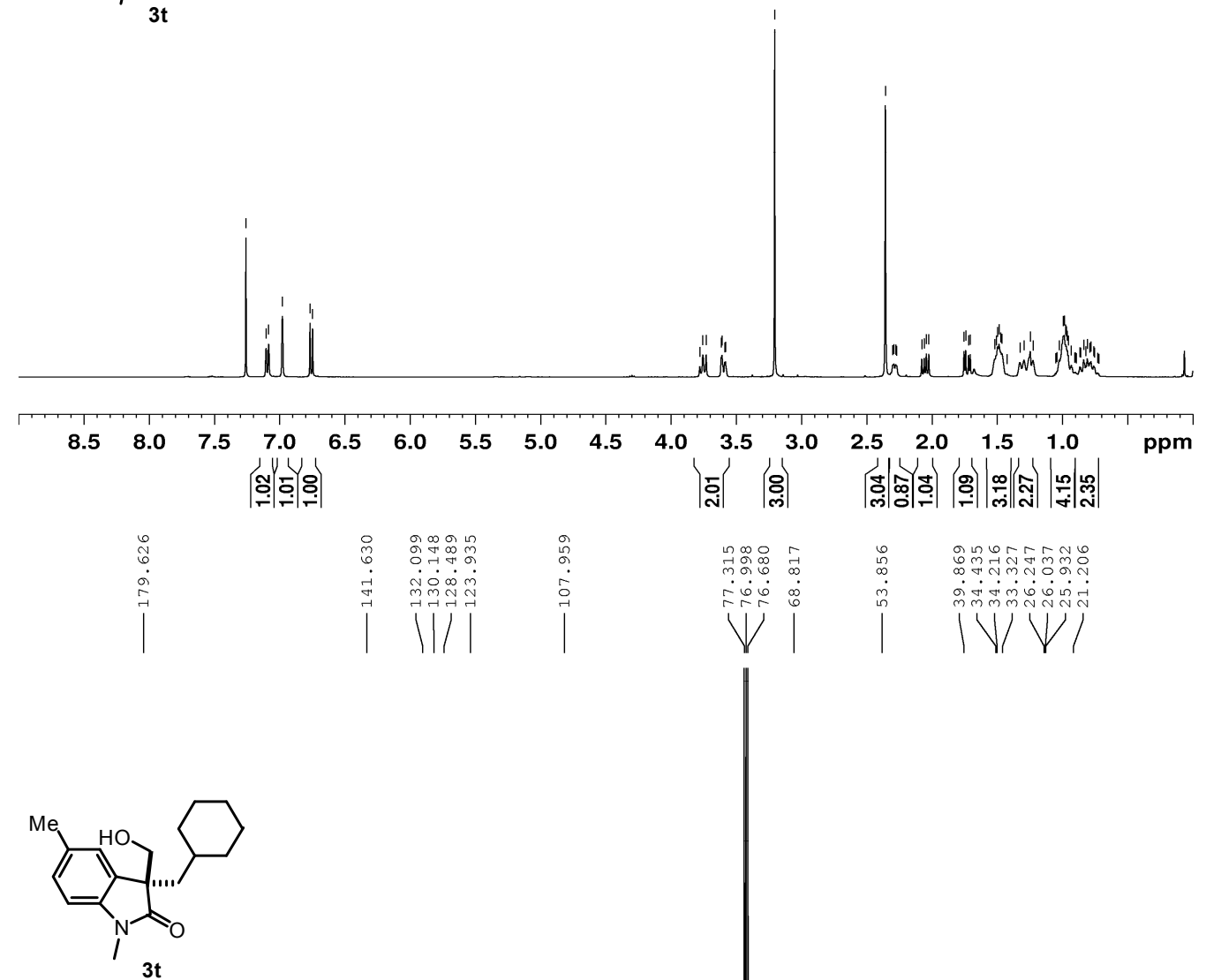

3t

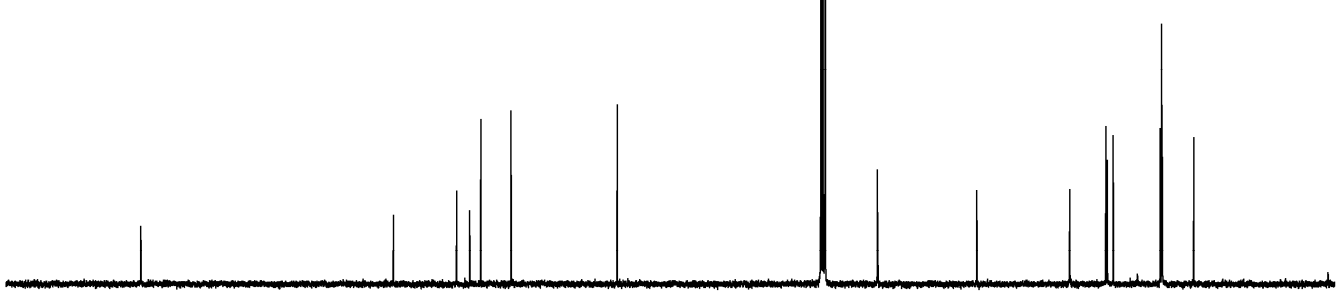

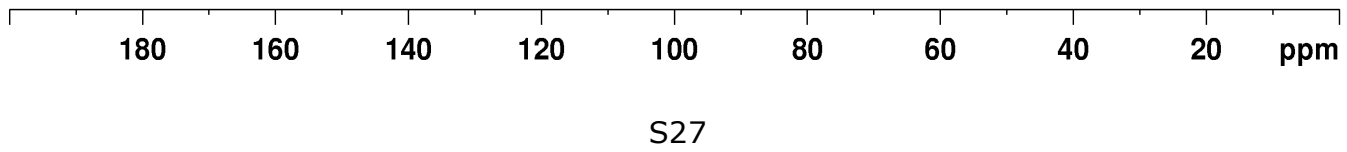




\section{${ }^{1} \mathrm{H}$ NMR and ${ }^{13} \mathrm{C}$ NMR Spectra of Products 4}
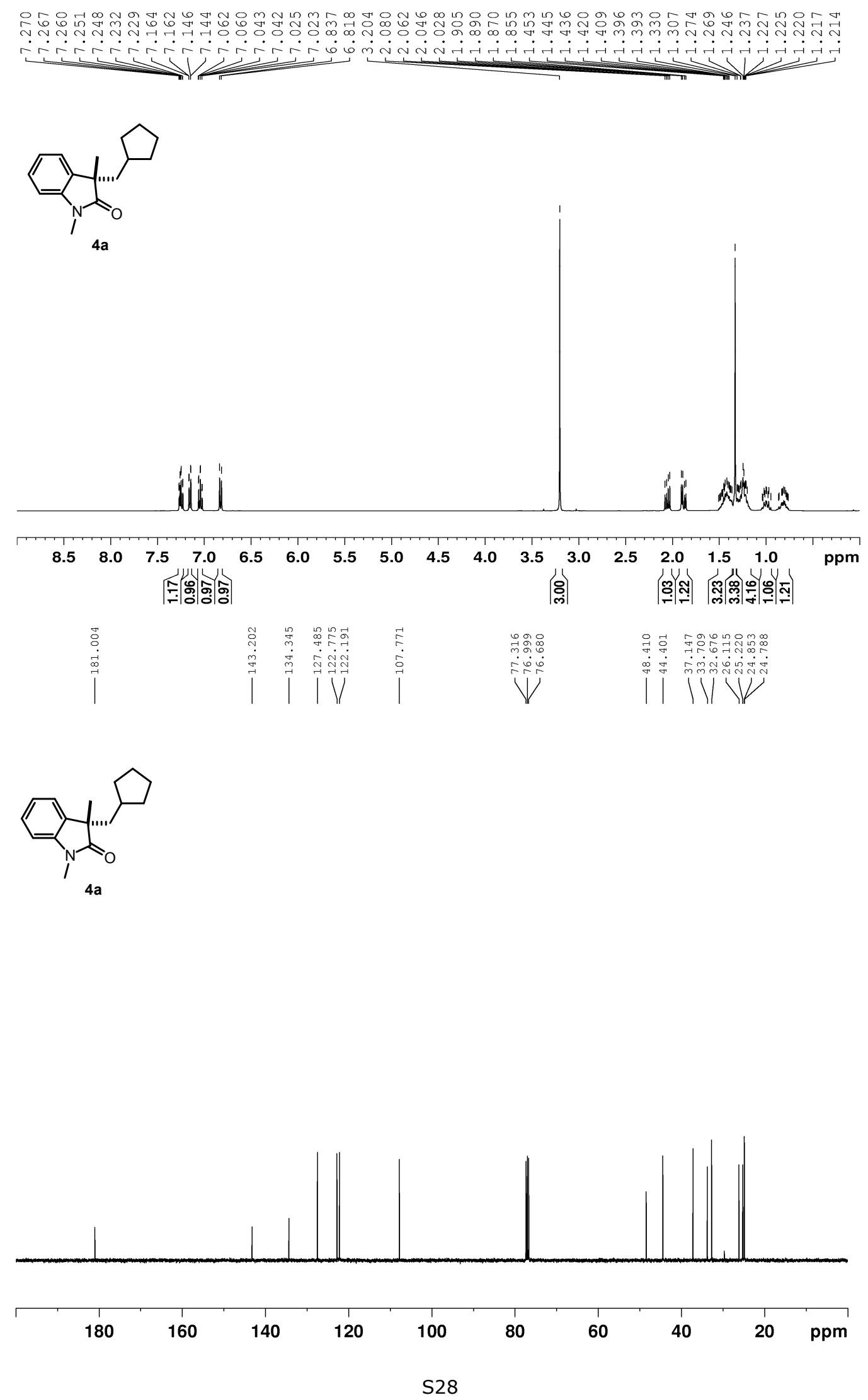

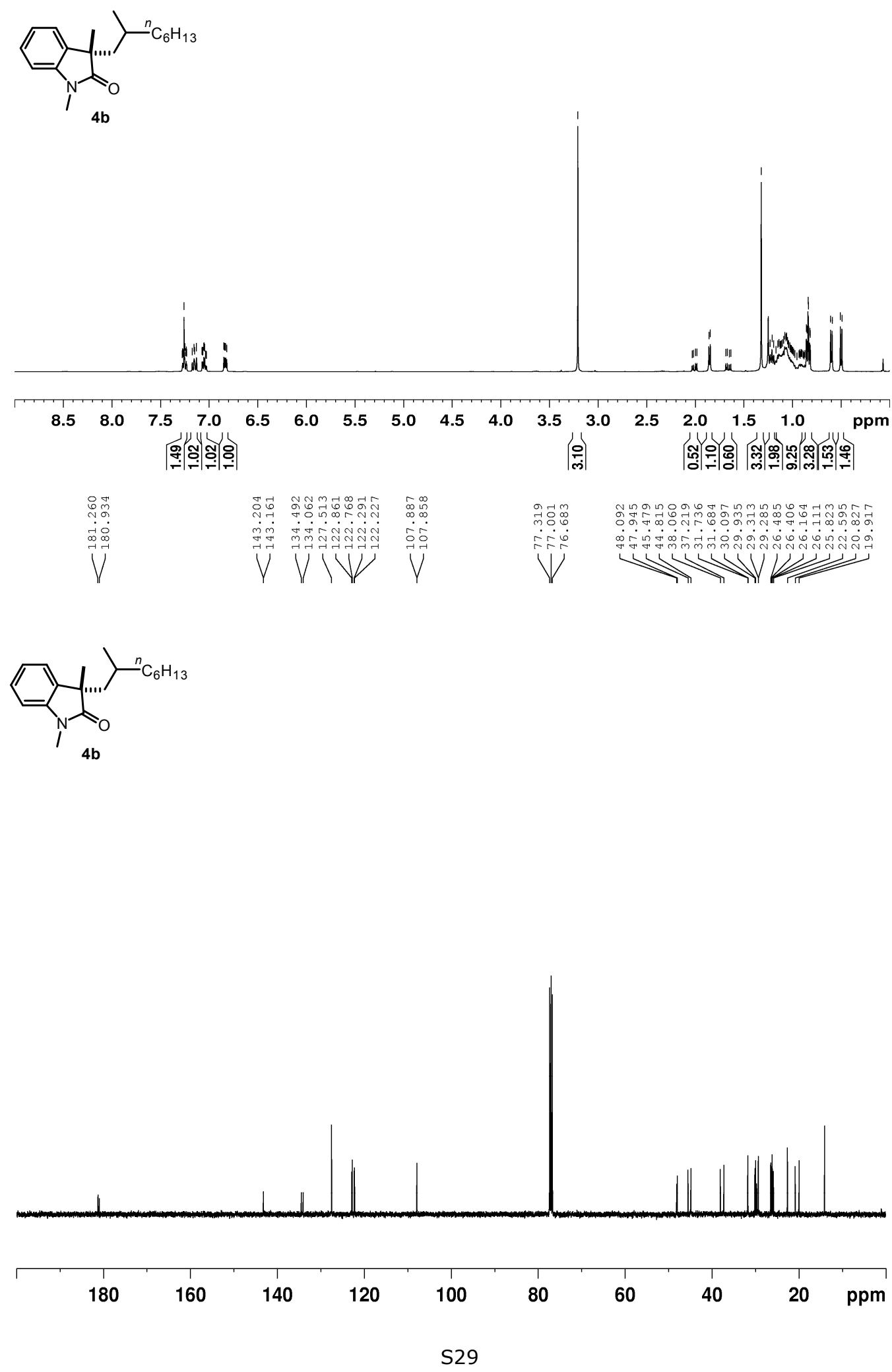


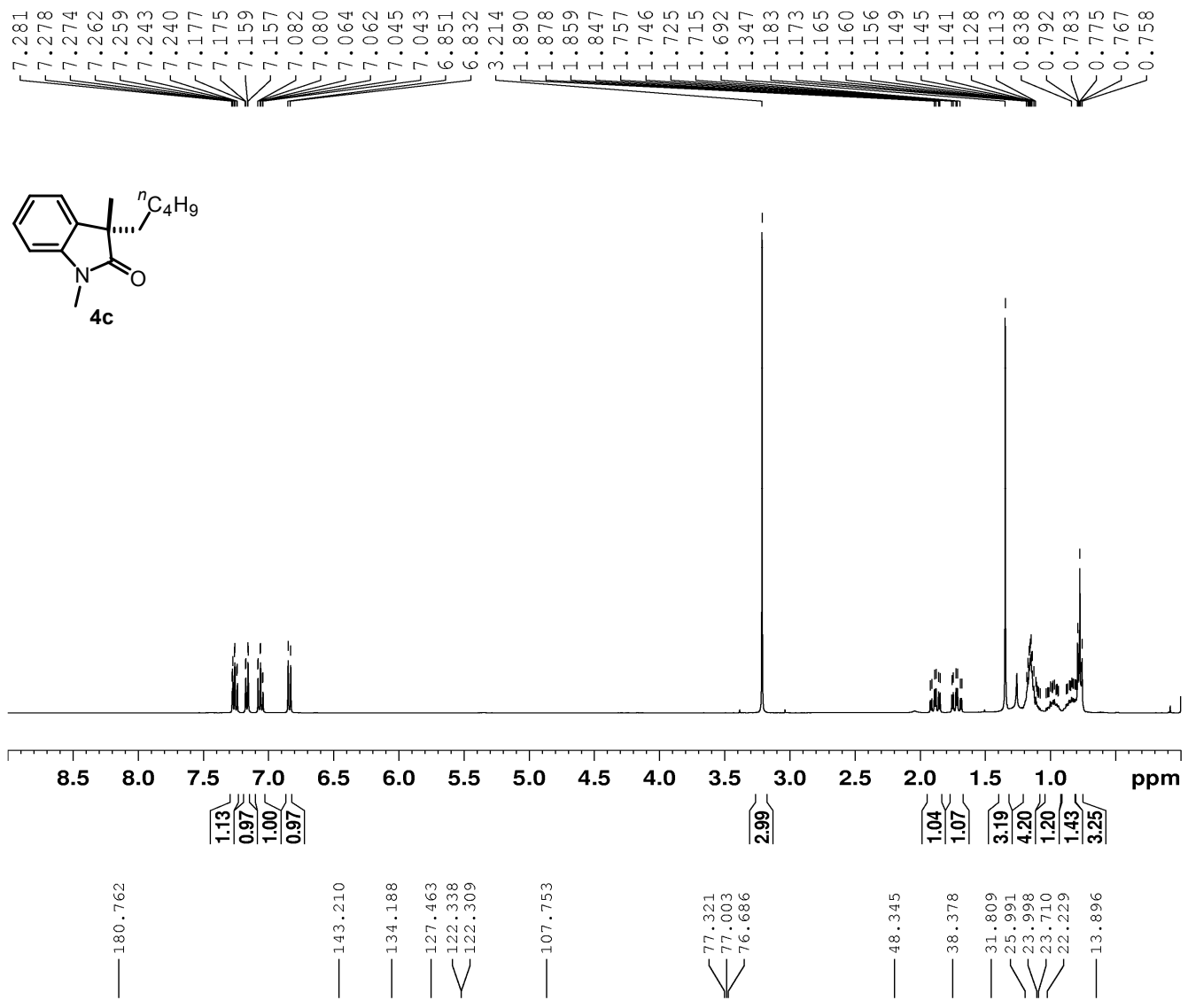

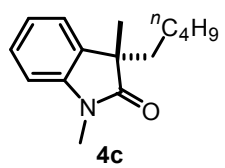
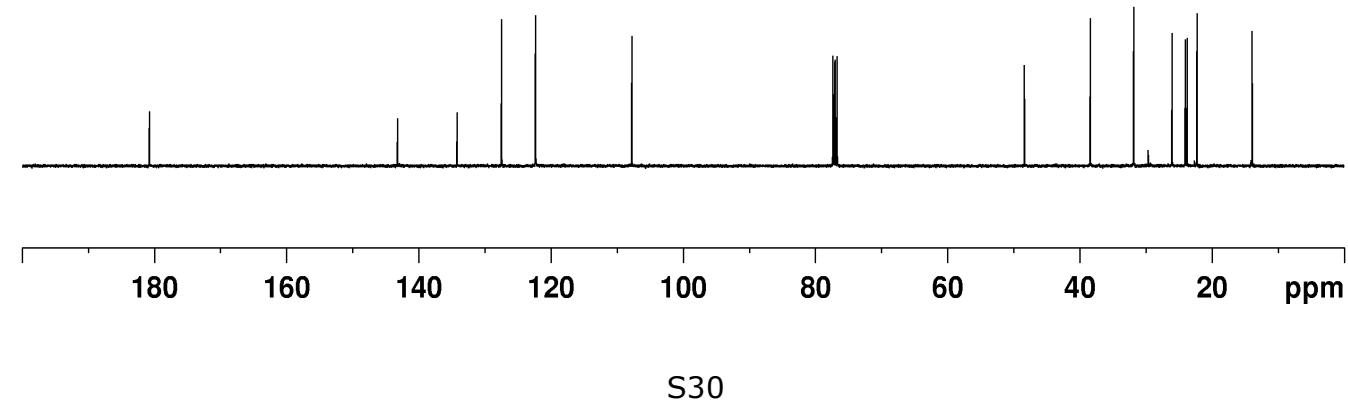


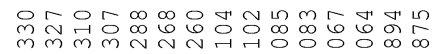

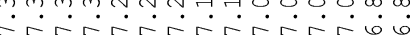

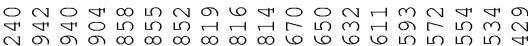

r.

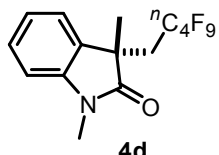

4d
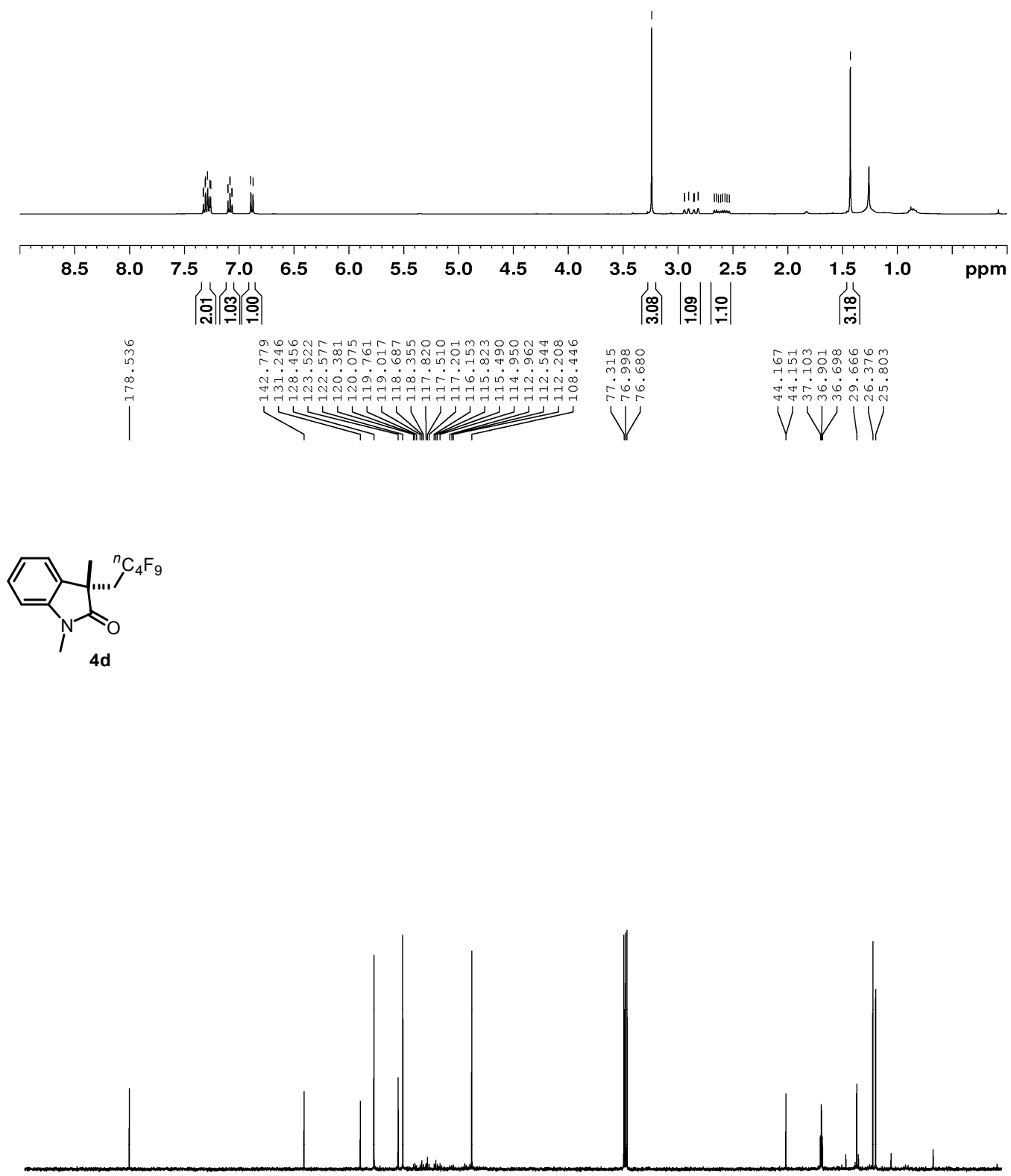

180

160

140

120

100

80

60

40 


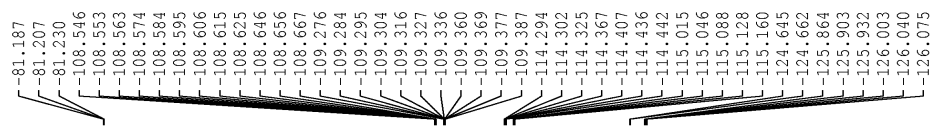

$\underbrace{n}_{i d}$

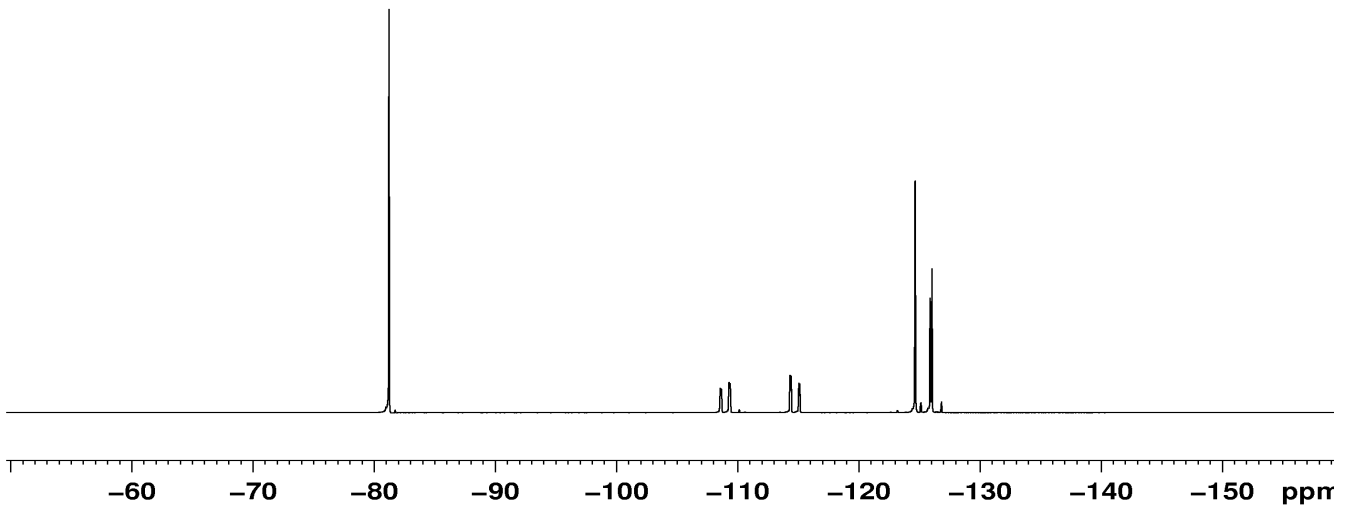




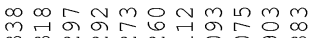

m.

inivisina

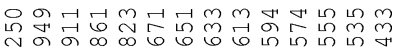

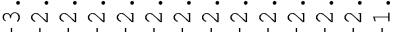

।
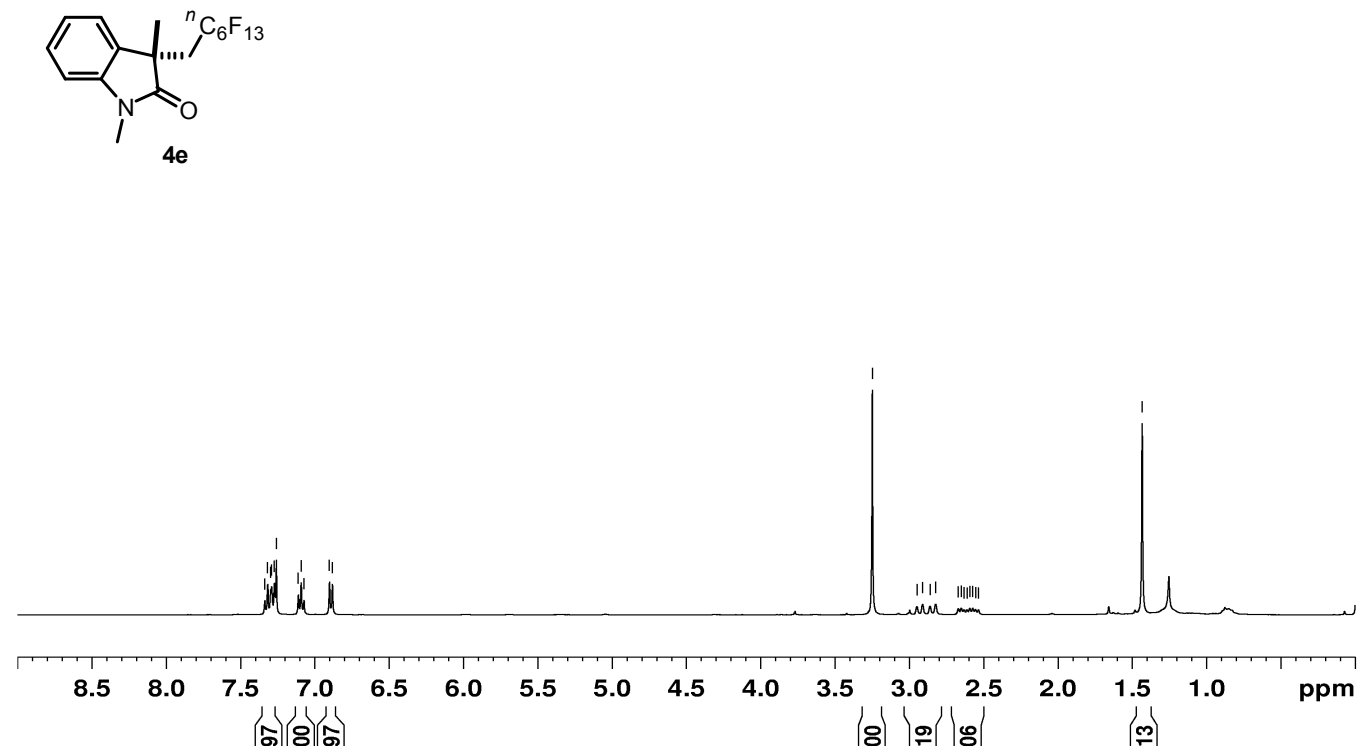
호유.

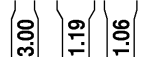

|m

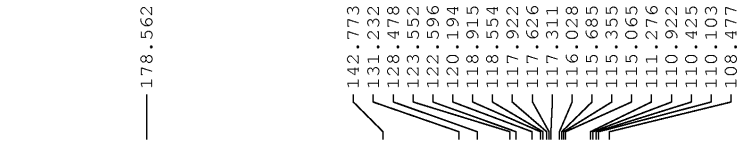

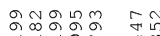

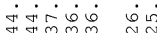

VV V

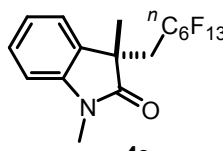

$4 e$

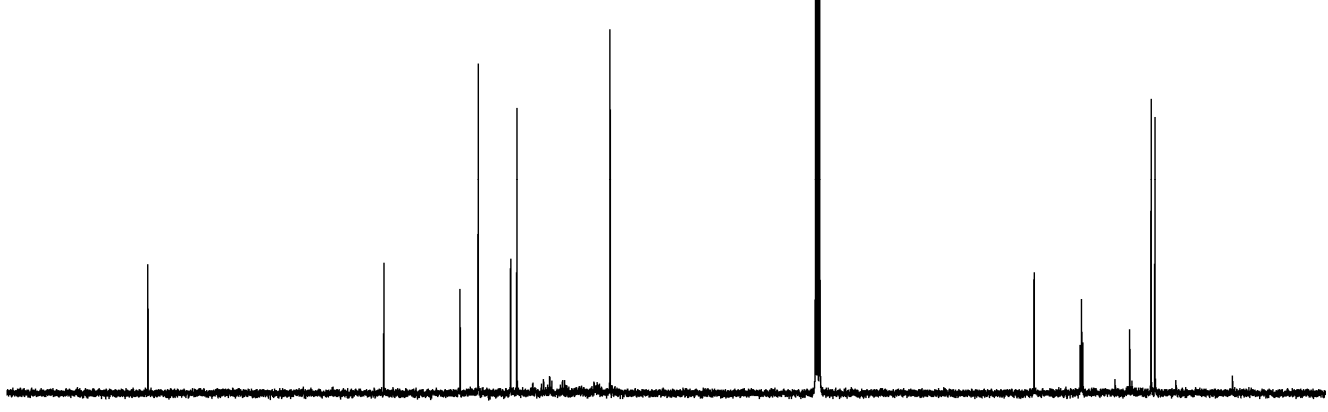

180

160

140

120

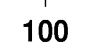

80

60

40

20 ppm 


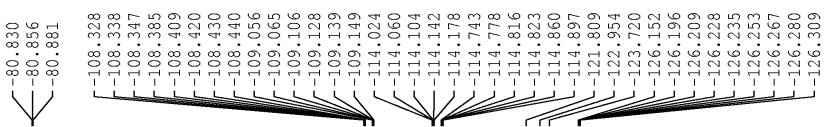

$\underbrace{n}_{i=0}$

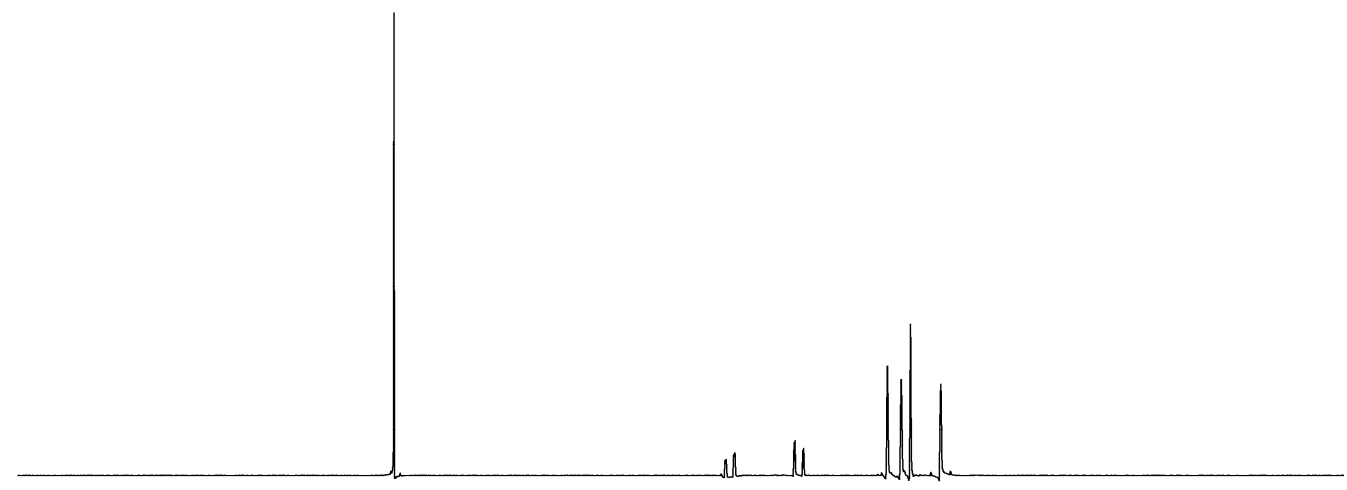

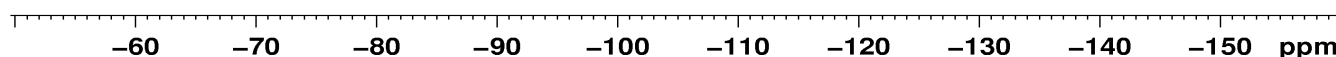



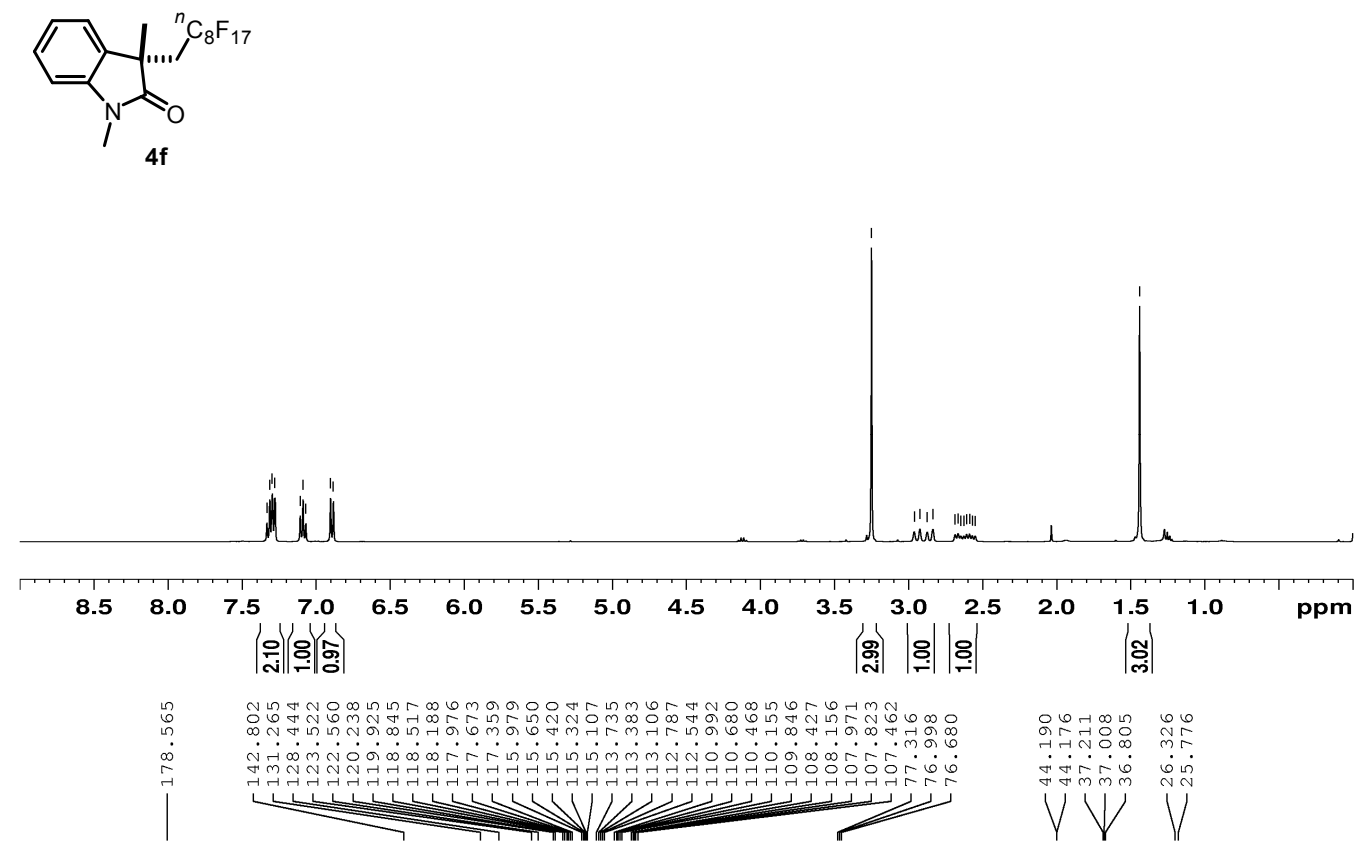

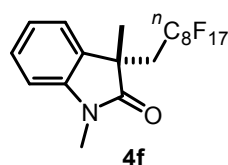
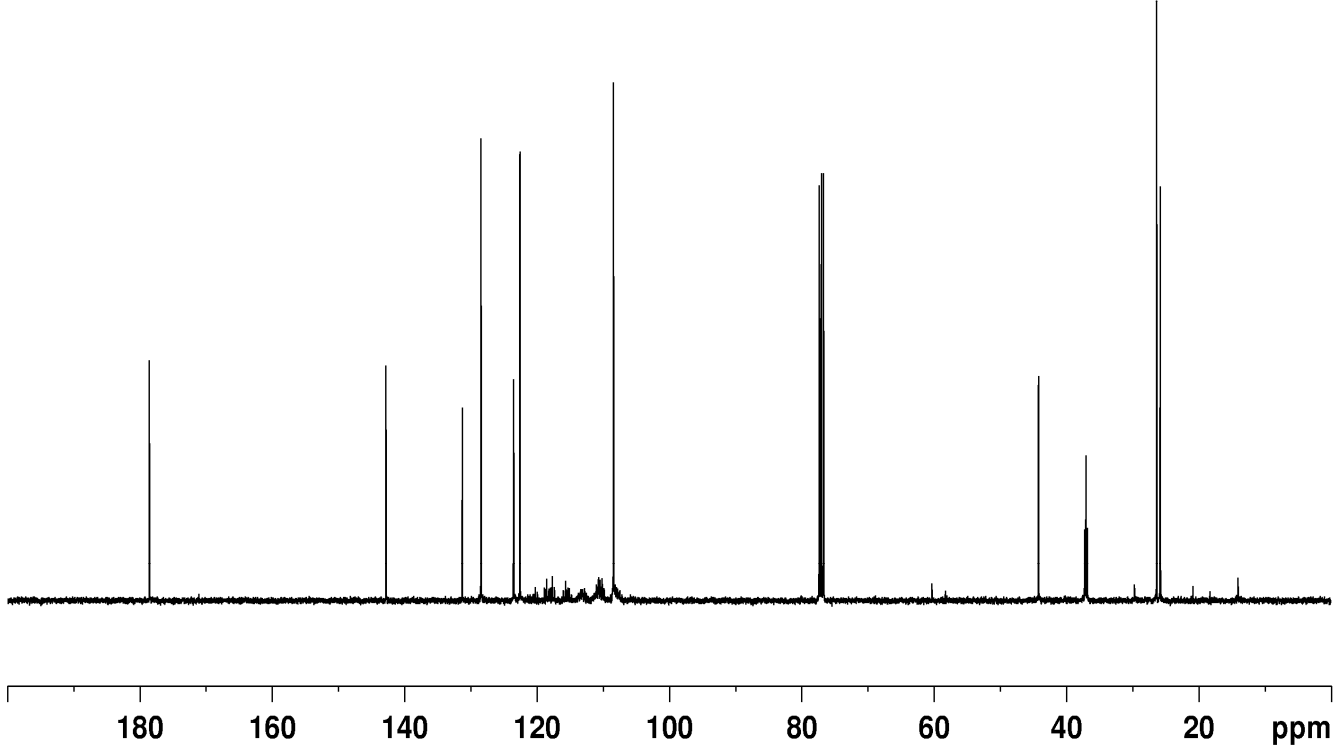


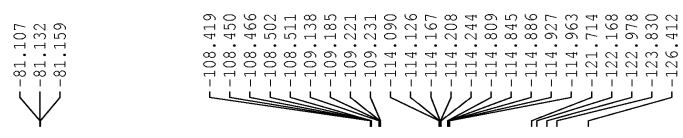

$\underbrace{}_{1 \mathrm{f}}{ }_{1}^{{ }^{n} \mathrm{C}_{8} \mathrm{~F}_{17}}$

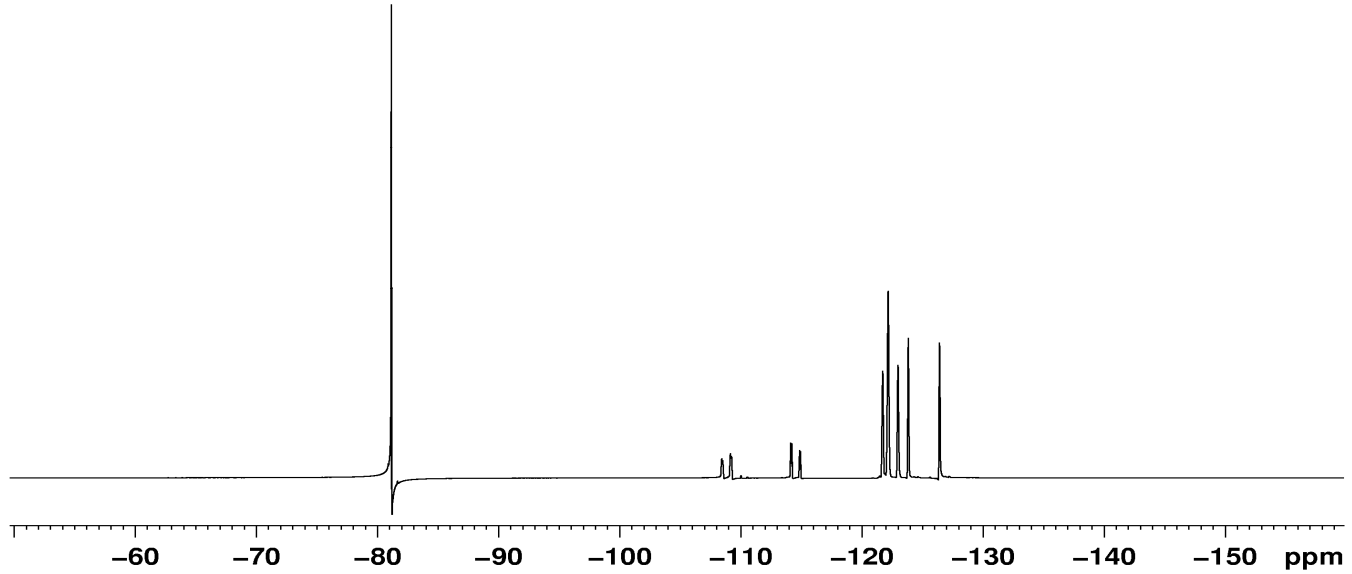



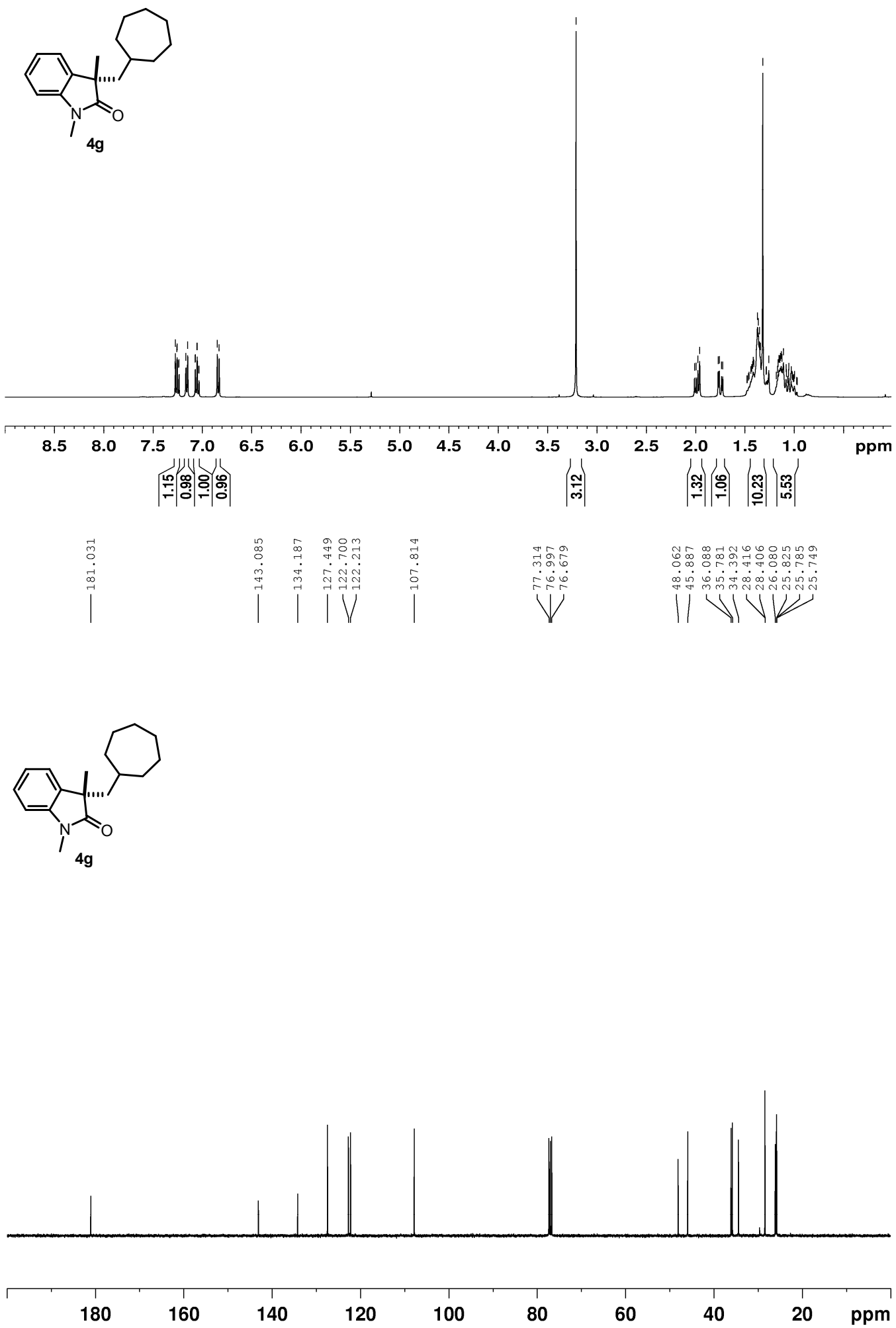
<smiles>CN1C(=O)[C@](C)(CBr)c2ccccc21</smiles>
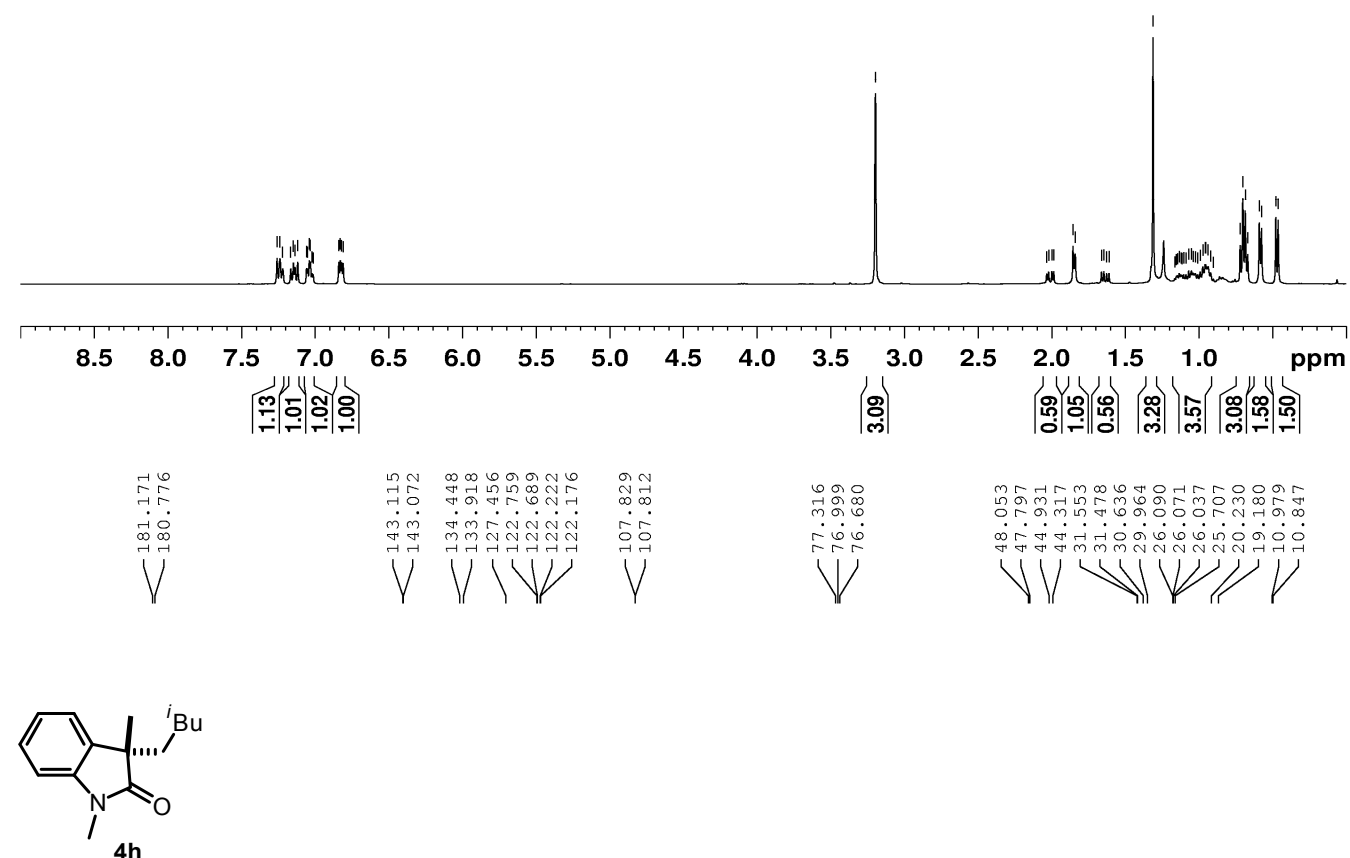

$4 \mathrm{~h}$
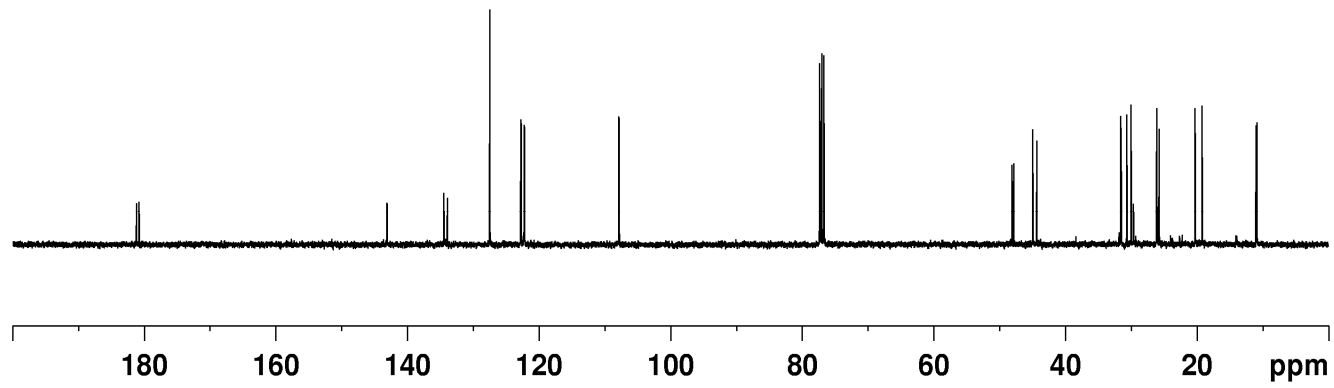


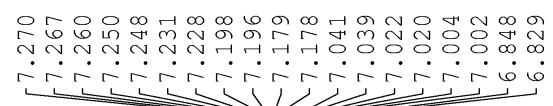

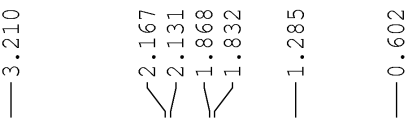

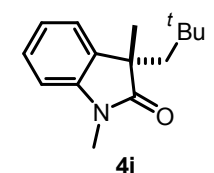

$4 i$

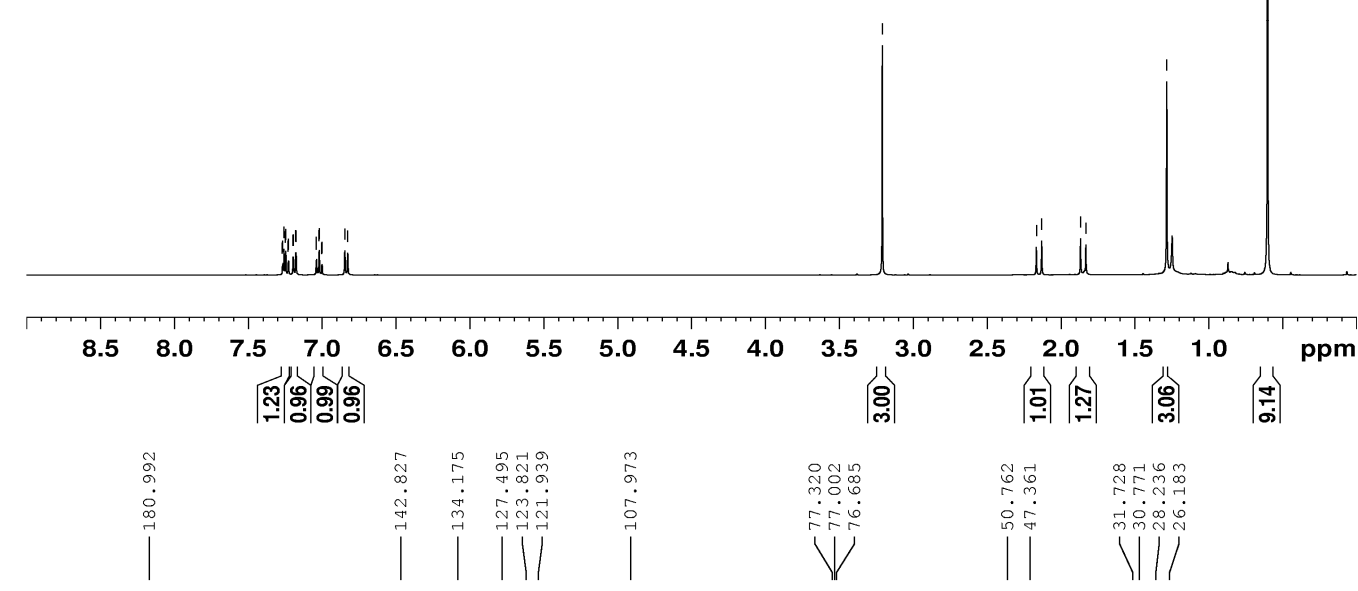

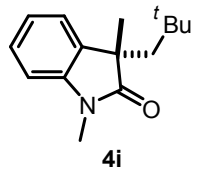

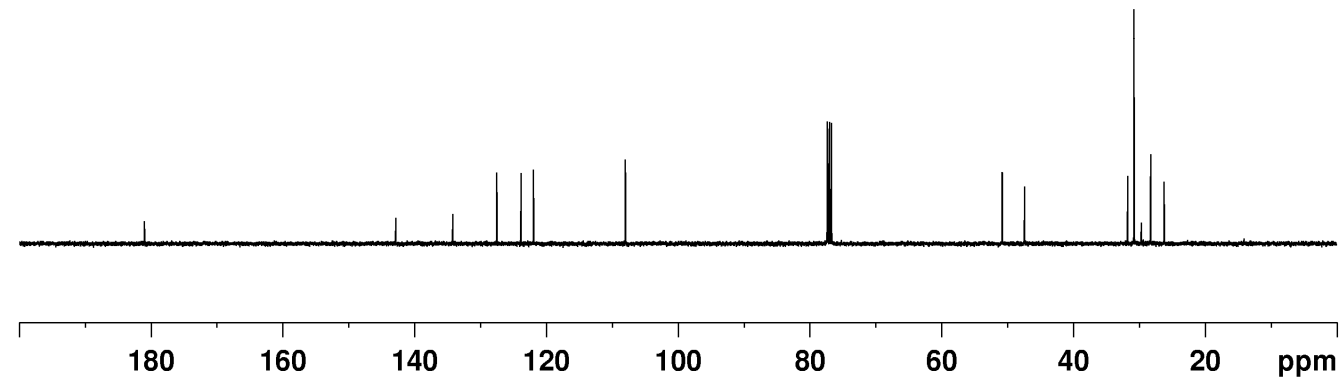



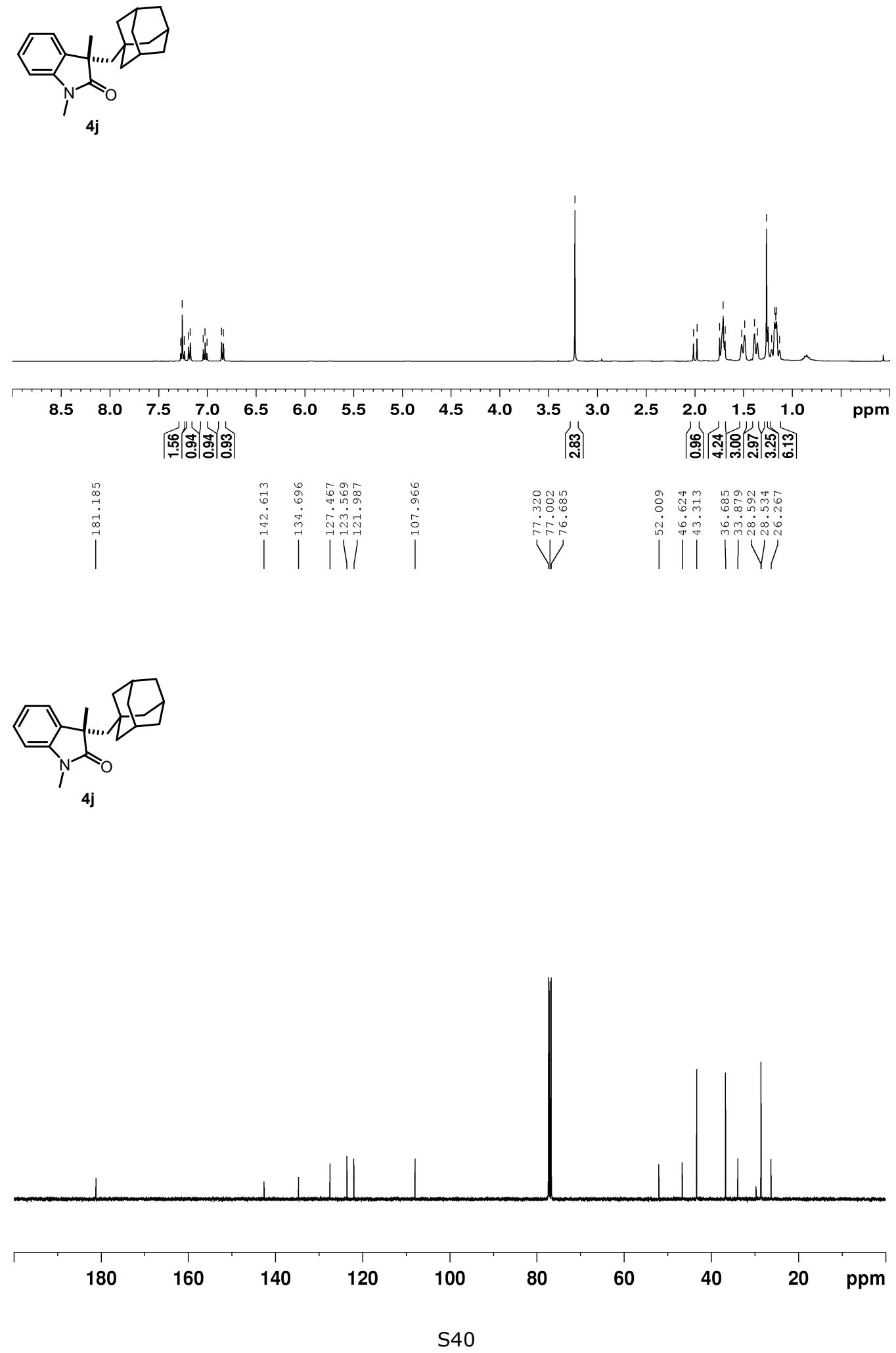

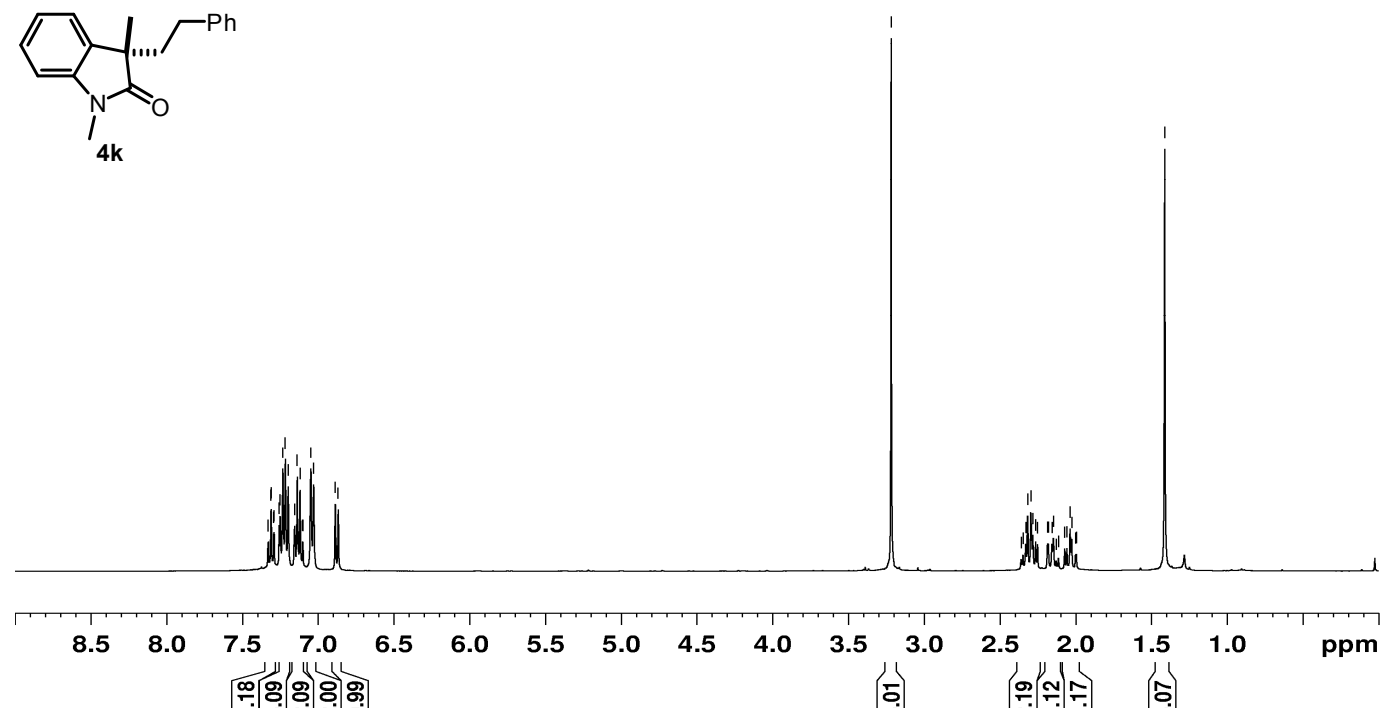

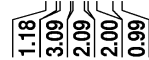

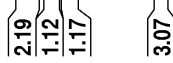
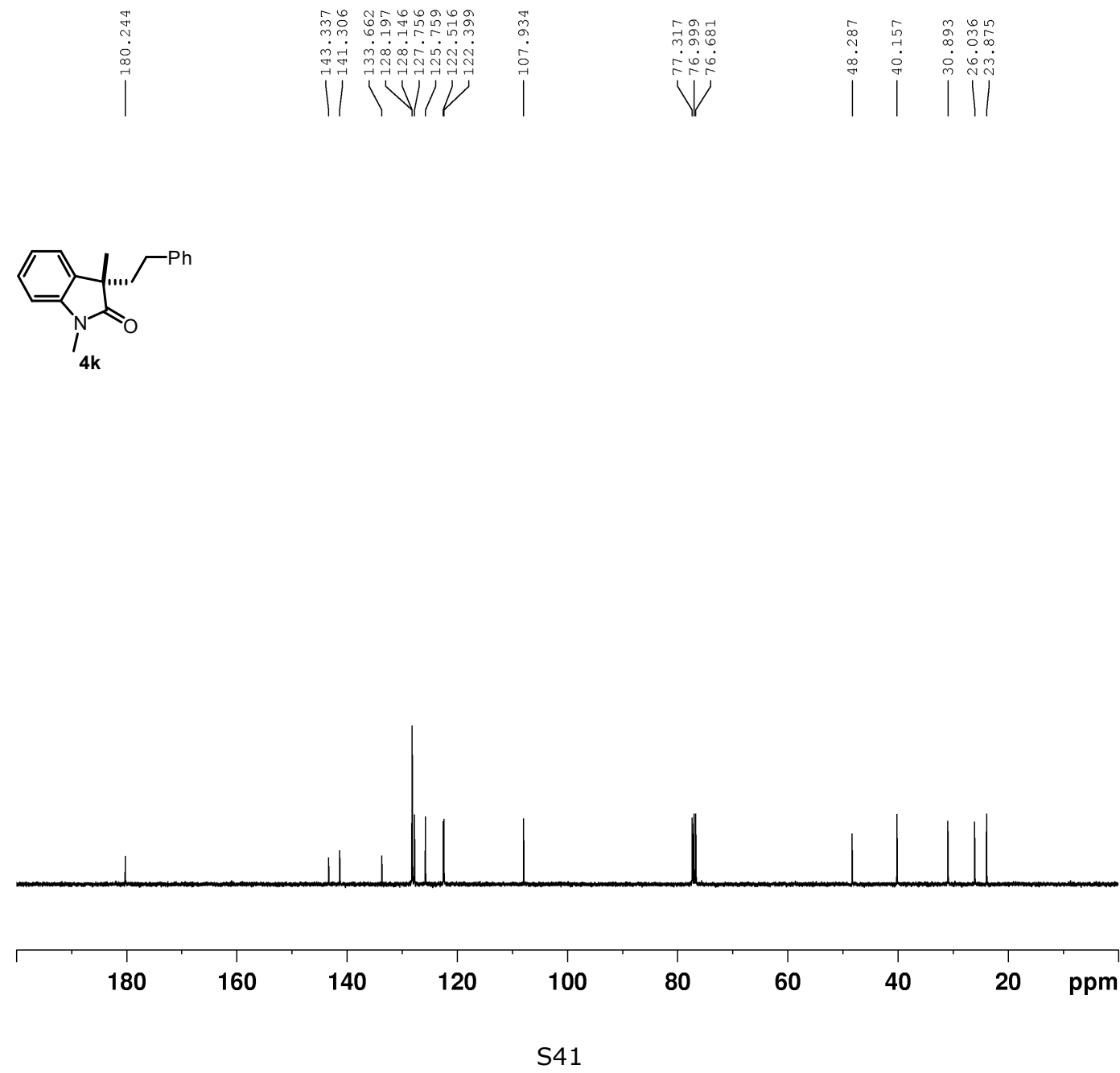

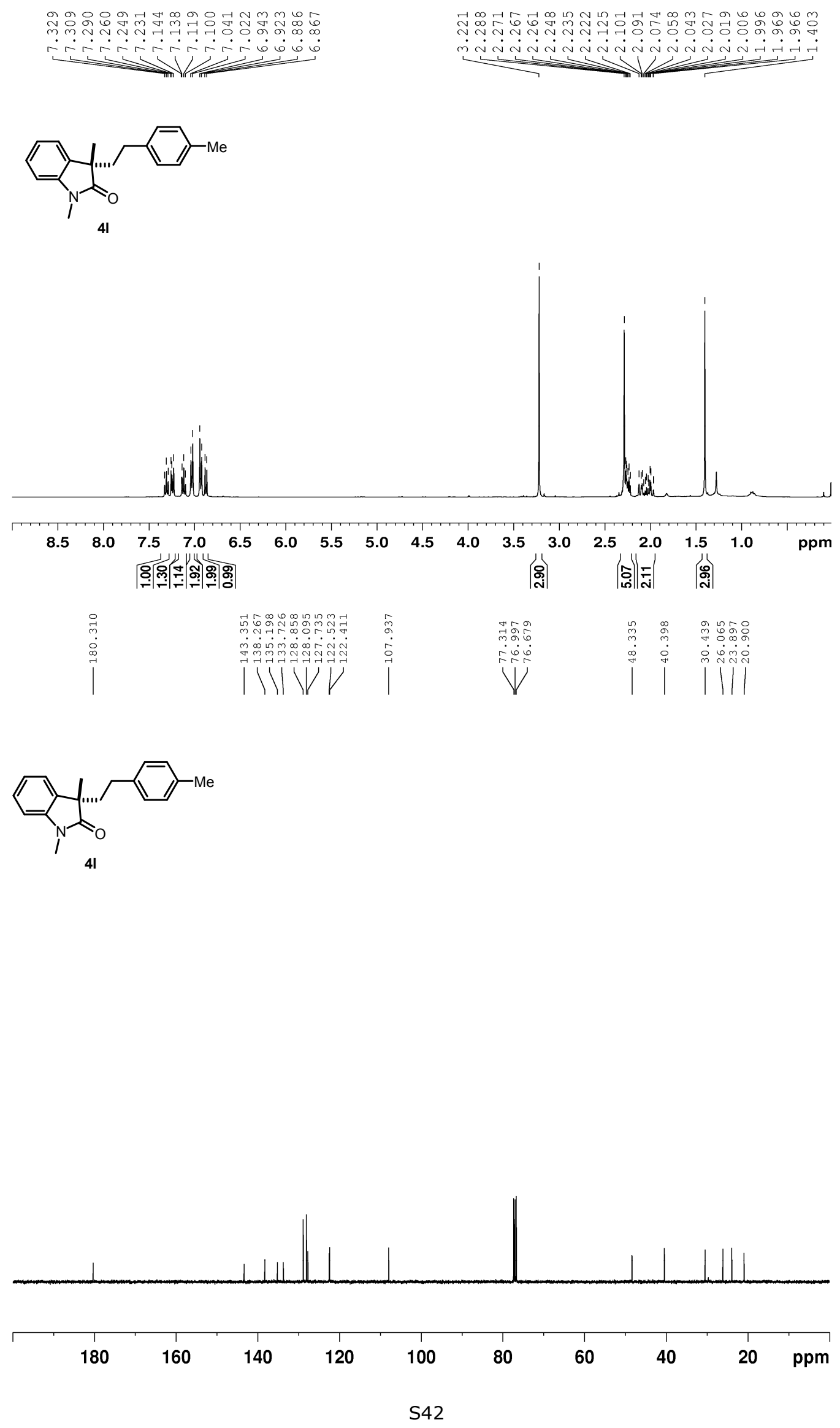


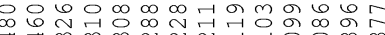

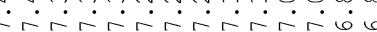

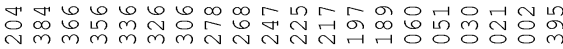

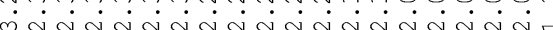
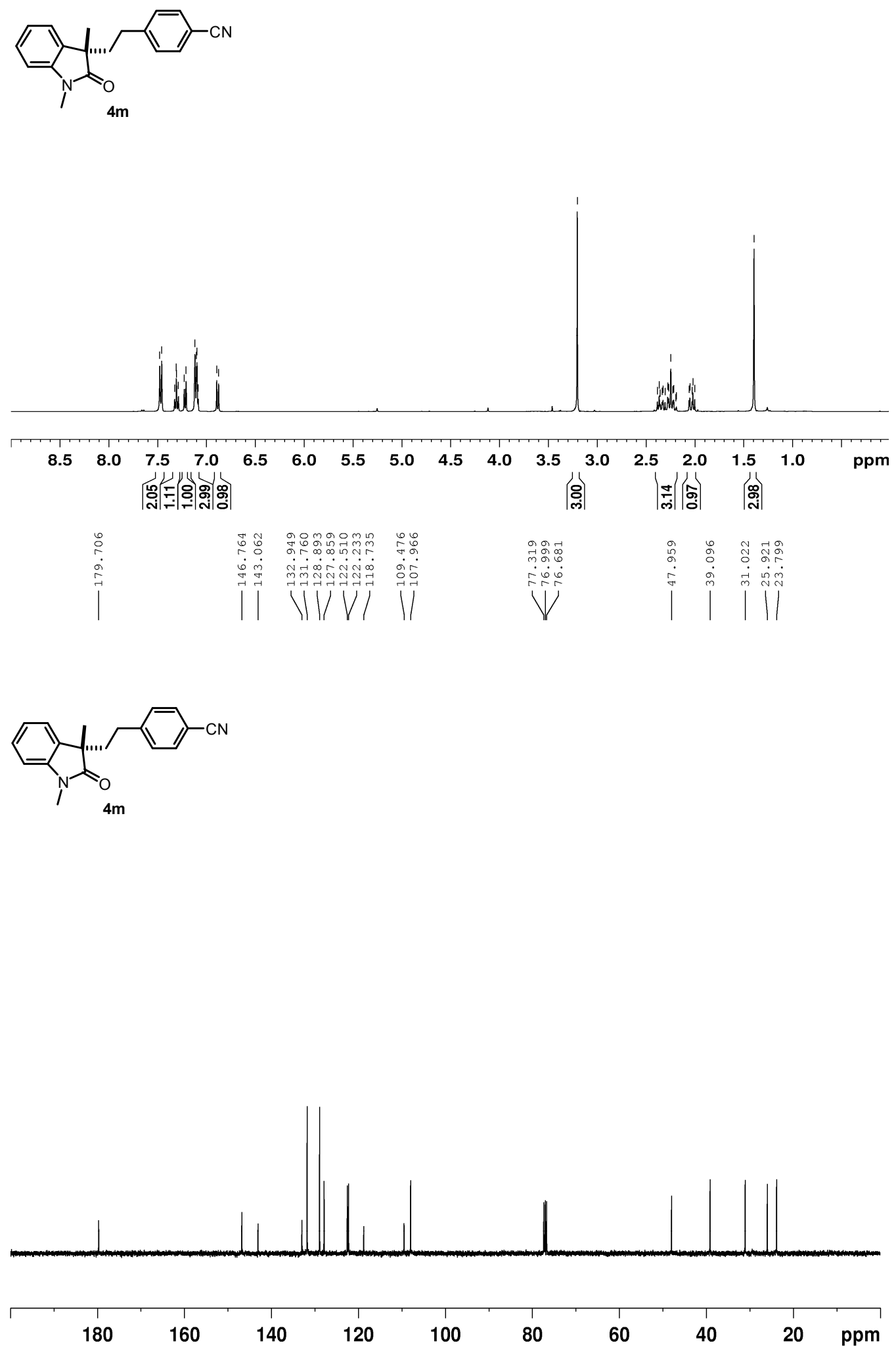


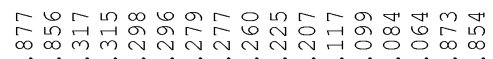

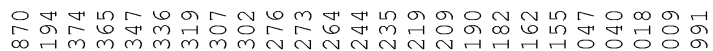

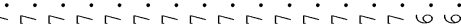

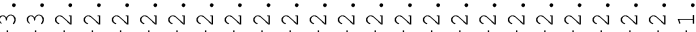

$\ldots$<smiles>CC(=O)c1ccc(CCC2(C)C(=O)N(C)c3ccccc32)cc1</smiles>

$4 n$

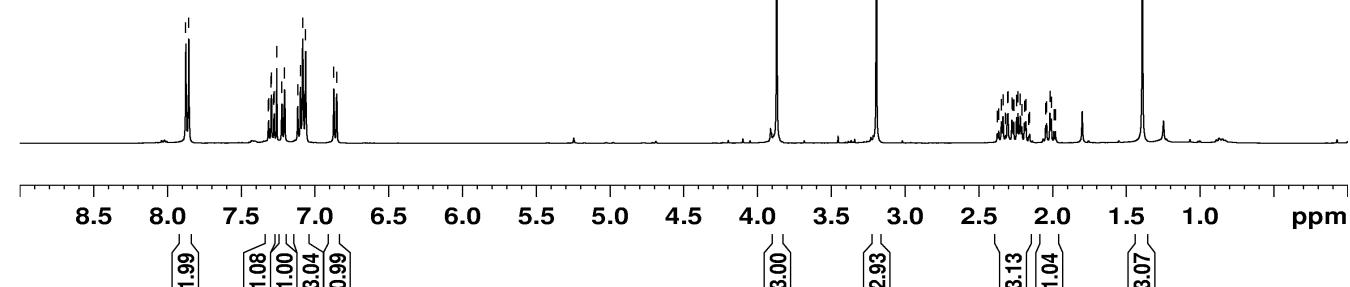

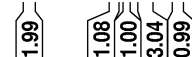
|⿱宀⿻心㇒日

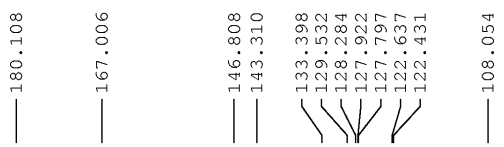

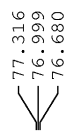
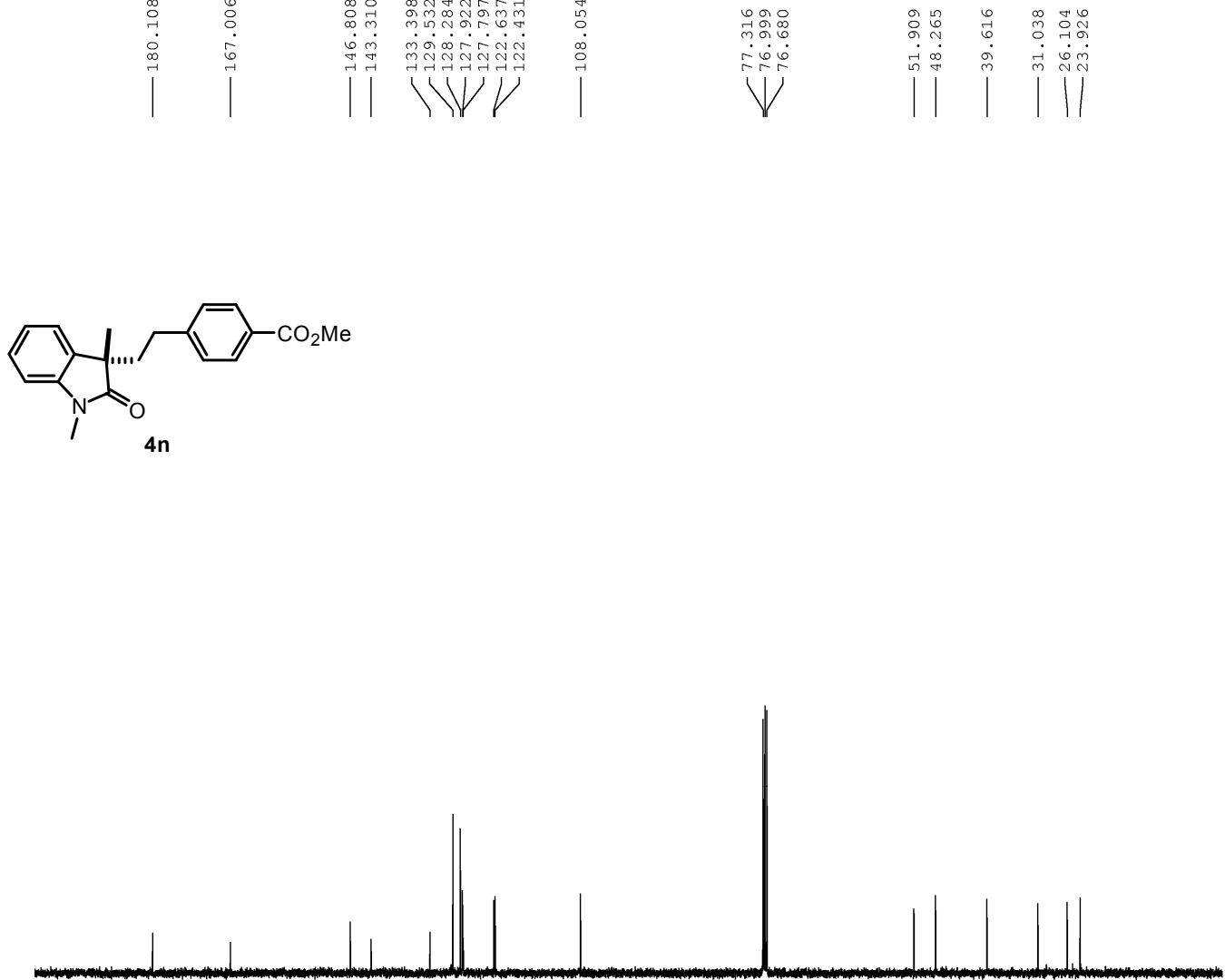

180

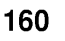

140

120

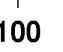

80

60

40

20 ppm 


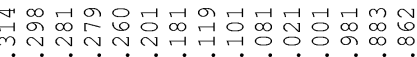

rariraririó

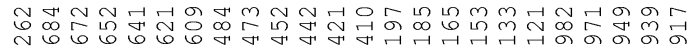<smiles>CN1C(=O)[C@](C)(CCc2c(Cl)cccc2Cl)c2ccccc21</smiles>
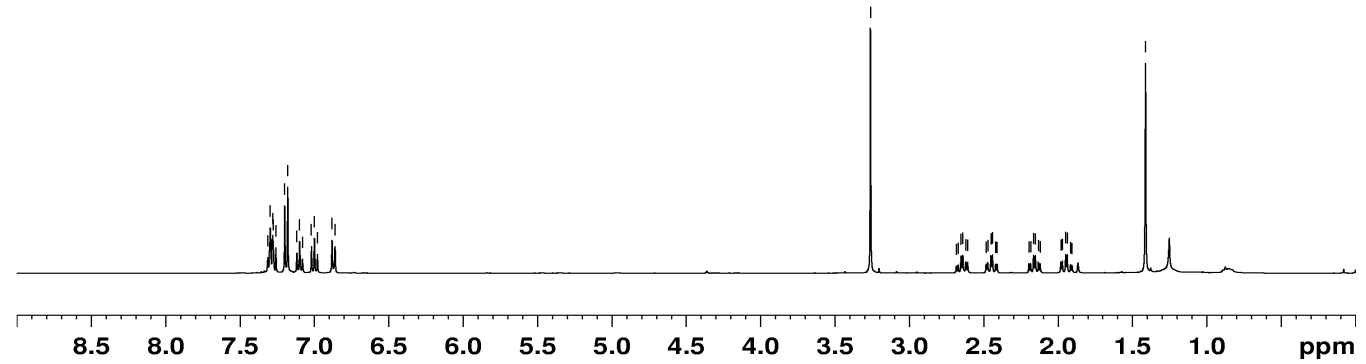

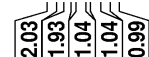

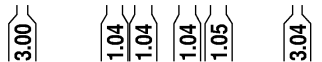

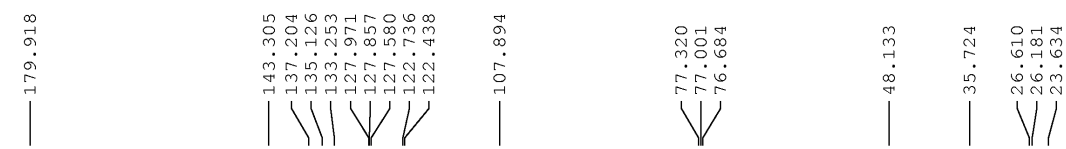<smiles>CN1C(=O)[C@](C)(CCc2c(Cl)cccc2Cl)c2ccccc21</smiles>

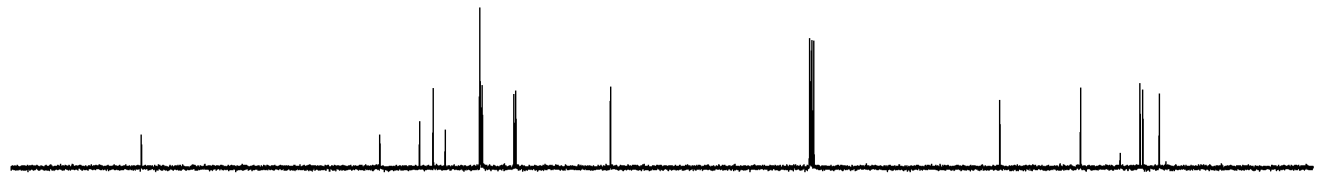

\begin{tabular}{|rrrrrrrrr|r|r|}
\hline 180 & 160 & 140 & 120 & 100 & 80 & 60 & 40 & 20 & ppm
\end{tabular}


<smiles>CN1C(=O)C(C2CCCCC2)C(c2ccccc2)c2ccccc21</smiles>

6a

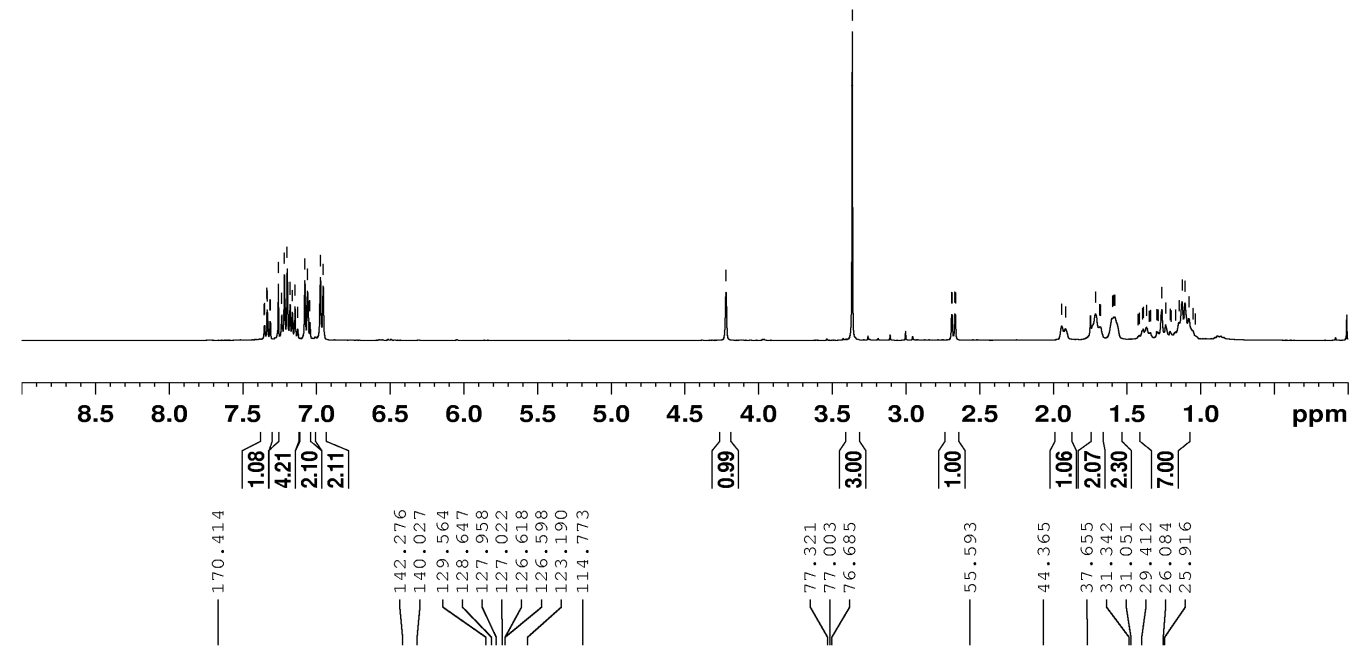<smiles>CN1C(=O)C(C2CCCCC2)C(c2ccccc2)c2ccccc21</smiles>

$6 a$
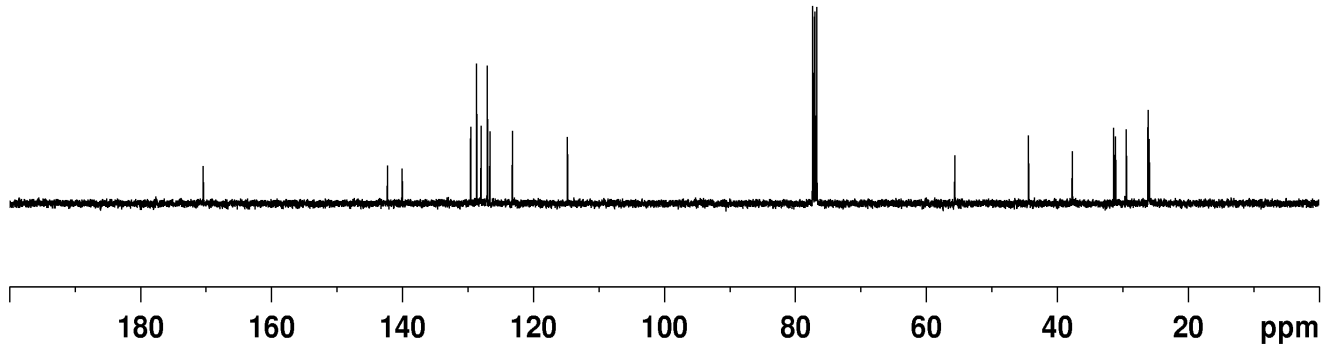
<smiles>C=CCCC[C@]1(C)C(=O)N(C)c2ccccc21</smiles>

$8 a$

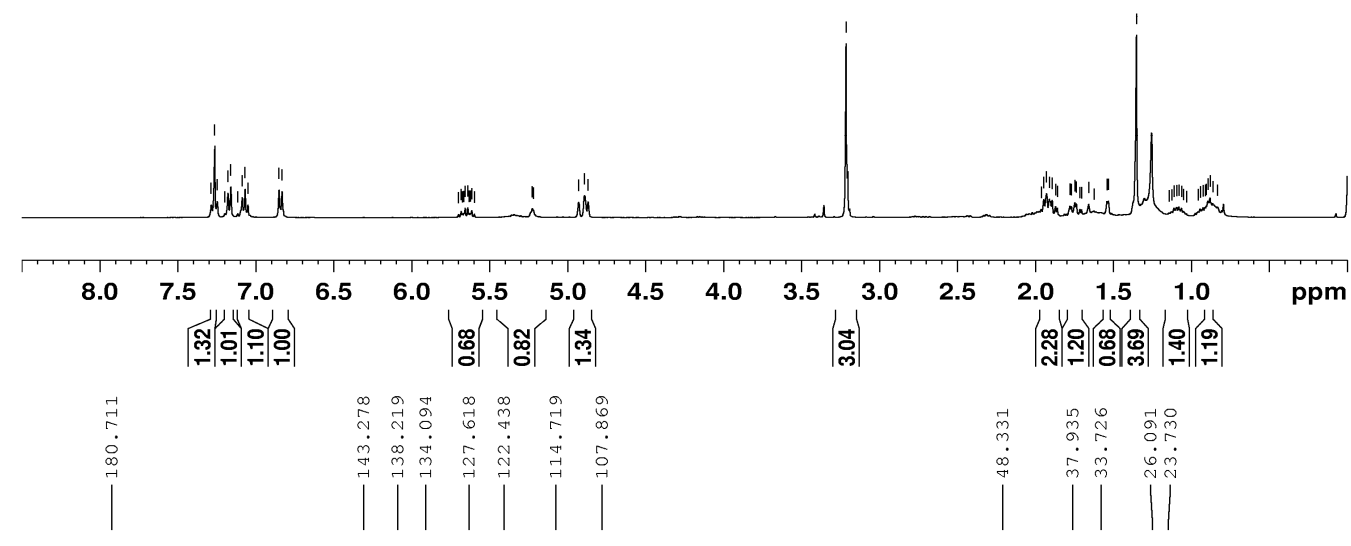<smiles>C=CCCCC1(C)C(=O)N(C)c2ccccc21</smiles>

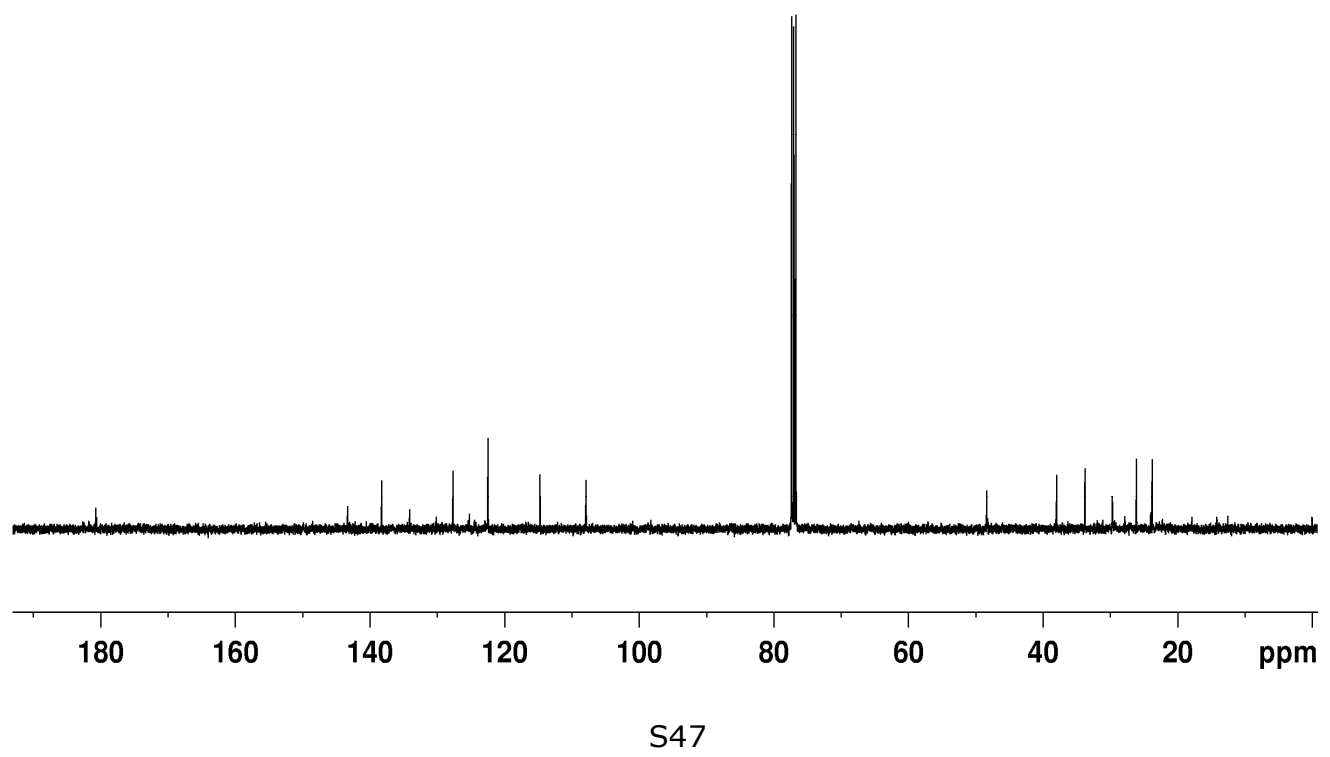




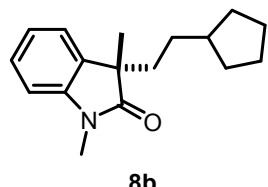

$8 b$
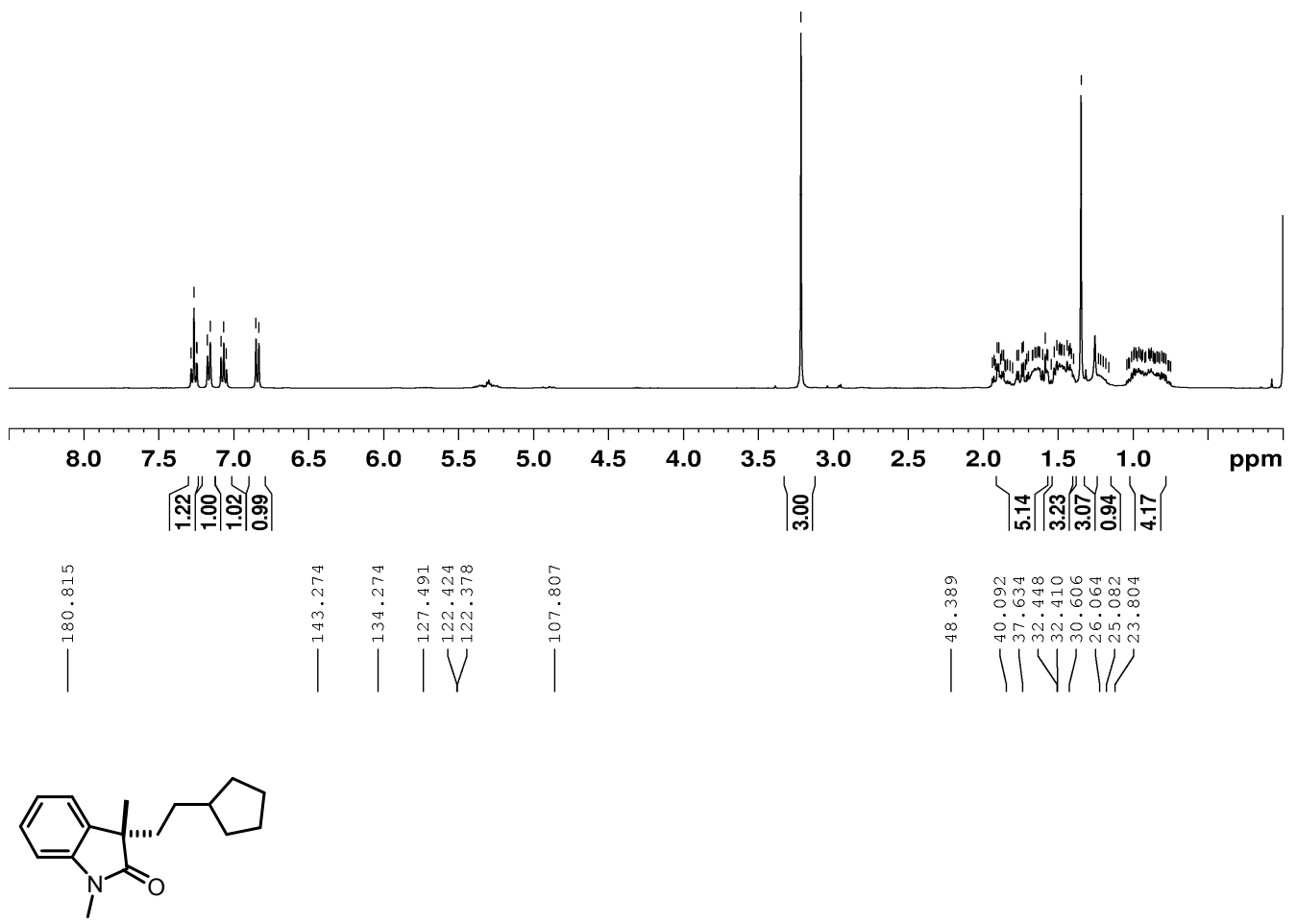

$8 b$

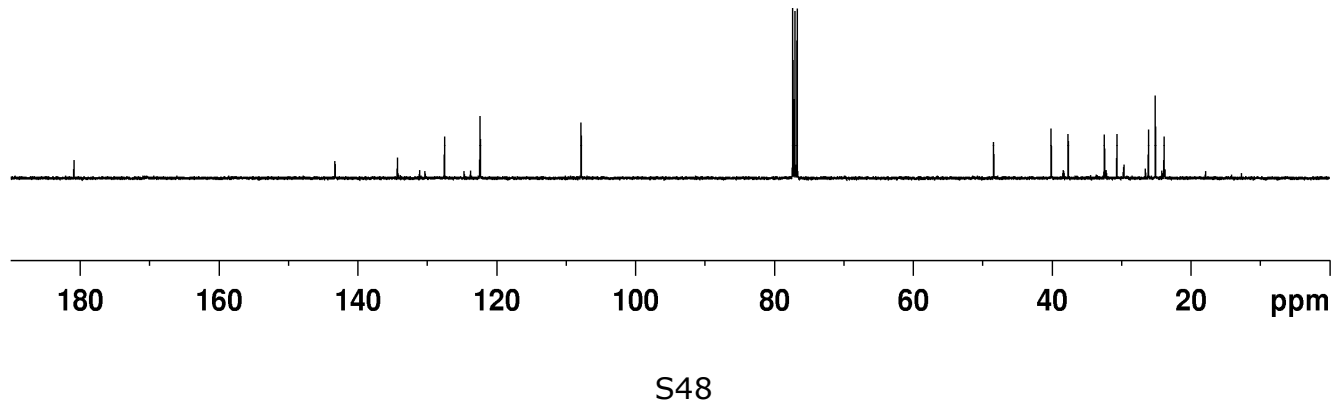

